\title{
EXTREME RESIDUAL DEPENDENCE FOR RANDOM VECTORS AND PROCESSES
}

\author{
LAURENS DE HAAN, ${ }^{*}$ Tilburg University and University of Lisbon \\ CHEN ZHOU,** De Nederlandsche Bank and Erasmus University Rotterdam
}

\begin{abstract}
A two-dimensional random vector in the domain of attraction of an extreme value distribution $G$ is said to be asymptotically independent (i.e. in the tail) if $G$ is the product of its marginal distribution functions. Ledford and Tawn (1996) discussed a form of residual dependence in this case. In this paper we give a characterization of this phenomenon (see also Ramos and Ledford (2009)), and offer extensions to higher-dimensional spaces and stochastic processes. Systemic risk in the banking system is treated in a similar framework.
\end{abstract}

Keywords: Asymptotic independence; extreme residual dependence; spectral measure

2010 Mathematics Subject Classification: Primary 62G20

\section{Introduction}

What is extreme residual dependence? Let us start by recalling the extreme value dependence in multivariate extreme value theory (EVT).

In a two-dimensional setup, we consider a random vector $\left(X_{1}, X_{2}\right)$ with distribution function $F$. Denote its independent and identically distributed (i.i.d.) copies as $\left(X_{1}^{(1)}, X_{2}^{(1)}\right)$, $\left(X_{1}^{(2)}, X_{2}^{(2)}\right), \ldots$ We take partial maxima for each marginal as $M_{i, n}=\max _{1 \leq j \leq n} X_{i}^{(j)}$ for $i=1,2$. Multivariate EVT assumes the following limit relation: there exist sequences of constants $a_{i, n}>0, b_{i, n} \in \mathbb{R}$ for $i=1,2$ and a distribution function $G$ with nondegenerate marginals, such that

$$
\lim _{n \rightarrow \infty} \mathrm{P}\left(\frac{M_{1, n}-b_{1, n}}{a_{1, n}} \leq x_{1}, \frac{M_{2, n}-b_{2, n}}{a_{2, n}} \leq x_{2}\right)=G\left(x_{1}, x_{2}\right)
$$

for all continuity points $\left(x_{1}, x_{2}\right)$ of $G$. Then, the distribution function $F$ is in the domain of attraction of a multivariate extreme value distribution $G$. Equivalently, there exists real-valued functions $a_{i}(t)>0$ and $b_{i}(t)$ for $i=1,2$ such that

$$
\lim _{t \rightarrow \infty} t \mathrm{P}\left(\frac{X_{1}-b_{1}(t)}{a_{1}(t)}>x_{1} \text { or } \frac{X_{2}-b_{2}(t)}{a_{2}(t)}>x_{2}\right)=-\log G\left(x_{1}, x_{2}\right)
$$

for all continuity points $\left(x_{1}, x_{2}\right)$ of $G$.

\footnotetext{
Received 11 August 2009; revision received 13 October 2010.

* Postal address: Department of Econometrics and Operations Research, Tilburg University, PO Box 90153, 5000LE Tilburg, The Netherlands. Email address: ldehaan@ese.eur.nl Supported in part by FCT project PTDC/MAT/112770/2009.

** Postal address: Economics and Research Division, De Nederlandsche Bank, PO Box 98, 1000AB Amsterdam, The Netherlands. Email address: c.zhou@dnb.nl
} 
Let $F_{i}, i=1,2$, be the marginal distribution functions of $F$. Suppose that $F_{i}$ is a continuous distribution function. Multivariate EVT shows that the necessary and sufficient condition for $F$ being in the domain of attraction can be separated into two parts: each marginal distribution $F_{i}$ belongs to the domain of attraction, as in univariate EVT with extreme value index $\gamma_{i}$ (for details on the univariate condition, see Theorem 1.1.6 of de Haan and Ferreira (2006)); and the dependence structure satisfies, given any $\left(x_{1}, x_{2}\right)$ for which $0<G_{0}\left(x_{1}, x_{2}\right)<1$,

$$
\lim _{t \rightarrow \infty} t\left(1-\mathrm{P}\left(\frac{1}{1-F_{1}\left(X_{1}\right)} \leq t x_{1}, \frac{1}{1-F_{2}\left(X_{2}\right)} \leq t x_{2}\right)\right)=-\log G_{0}\left(x_{1}, x_{2}\right),
$$

where $G_{0}\left(x_{1}, x_{2}\right)=G\left(\left(x_{1}^{\gamma_{1}}-1\right) / \gamma_{1},\left(x_{2}^{\gamma_{2}}-1\right) / \gamma_{2}\right)$. Define $\tilde{X}_{i}=1 /\left(1-F_{i}\left(X_{i}\right)\right)$ for $i=1,2$. Then the marginal distributions of $\left(\tilde{X}_{1}, \tilde{X}_{2}\right)$ are both standard Pareto distributions, i.e. $\mathrm{P}\left(\tilde{X}_{i}>x\right)$ $=1 / x$ for $x>1$, which does not contain marginal information of $\left(X_{1}, X_{2}\right)$. Hence, relation (1.2) based on $\left(\tilde{X}_{1}, \tilde{X}_{2}\right)$ is a condition only on the extreme dependence of $\left(X_{1}, X_{2}\right)$. Thus, $G_{0}(x, y)$ characterizes the structure of the extreme value dependence.

The extreme value dependence can be further decomposed as follows. Condition (1.2) holds if and only if there exists a measure $v$ on $\mathbb{R}_{+}^{2}$ such that, for $x_{1}, x_{2}>0$,

$$
-\log G_{0}\left(x_{1}, x_{2}\right)=v\left\{(u, v): u>x_{1} \text { or } v>x_{2}\right\} .
$$

Then, for any Borel set $A \subset \mathbb{R}_{+}^{2}$ with $\inf _{\left(x_{1}, x_{2}\right) \in A} x_{1} \vee x_{2}>0$ and any $a>0$,

$$
v(a A)=a^{-1} v(A) .
$$

The measure $v$ is called the exponent measure. It has the following representation: there exists a probability measure $H$ on $[0,1]$ with mean $\frac{1}{2}$, such that

$$
v\left\{(u, v): u>x_{1} \text { or } v>x_{2}\right\}=2 \int_{0}^{1} \frac{w}{x_{1}} \vee \frac{1-w}{x_{2}} H(\mathrm{~d} w) .
$$

The measure $H$ is called the spectral measure. The limiting distribution $G_{0}$ in (1.2) is determined by either $v$ or $H$.

Conversely, any exponent measures $v$ satisfying (1.4) or any probability measures $H$ on $[0,1]$ with mean $\frac{1}{2}$ occur as in (1.2)-(1.5). This can be seen by choosing

$$
U_{1}=2 R \Theta, \quad U_{2}=2 R(1-\Theta),
$$

where $R$ and $\Theta$ are independent random variables with distribution functions $\mathrm{P}(R>r)=\frac{1}{r}$ for $r>1$ and $\mathrm{P}(\Theta \leq w)=H(w)$ for $w \in[0,1]$. To see this, firstly check that $F_{U_{i}}(x):=$ $\mathrm{P}\left(U_{i} \leq x\right)=1-1 / x$ for $x>2$ and $i=1,2$. Thus, $1 /\left(1-F_{U_{i}}(x)\right)=x$ for $x>2$. Next, check that, for any $x_{1}, x_{2}>0$,

$$
\lim _{t \rightarrow \infty} t \mathrm{P}\left(2 R \Theta>t x_{1} \text { or } 2 R(1-\Theta)>t x_{2}\right)=2 \int_{0}^{1} \frac{w}{x_{1}} \vee \frac{1-w}{x_{2}} H(\mathrm{~d} w) .
$$

Then it is obvious that the random vector $\left(U_{1}, U_{2}\right)$ belongs to the domain of attraction with spectral measure $H$. For details, see de Haan and Resnick (1977) and de Haan and Ferreira (2006, Chapter 6).

A special case occurs when the spectral measure $H$ is concentrated on the points $\{0\}$ and $\{1\}$ with measure $\frac{1}{2}$ each. In that case,

$$
v\left\{(u, v): u>x_{1} \text { or } v>x_{2}\right\}=\frac{1}{x_{1}}+\frac{1}{x_{2}}=v\left\{(u, v): u>x_{1}\right\}+v\left\{(u, v): v>x_{2}\right\}
$$

for $x_{1}, x_{2}>0$, i.e. the exponent measure $v$ is concentrated on the coordinates. This additive 
property translates into a product property for the limit distribution function $G_{0}$ in (1.2), i.e. the distribution function $G_{0}$, hence $G$, is the product of two marginal distributions. This phenomenon is called asymptotic independence (cf. Geffroy (1958) and Sibuya (1960)). Furthermore, we obtain

$$
\lim _{t \rightarrow \infty} t \mathrm{P}\left(\tilde{X}_{1}>t x_{1} \text { and } \tilde{X}_{2}>t x_{2}\right)=v\left\{(u, v): u>x_{1} \text { and } v>x_{2}\right\}=0
$$

for $x_{1}, x_{2}>0$. Hence, no asymptotic information about sets of the form $\left\{(u, v): u>x_{1}\right.$ and $\left.v>x_{2}\right\}$ is obtained.

Ledford and Tawn in a series of papers (see Ledford and Tawn (1996), (1997), (1998), and (2003)) filled in the gap by introducing the additional natural assumption that, for some $0<\eta<1$ and all $x_{1}, x_{2}>0, \mathrm{P}\left(\tilde{X}_{1}>t x_{1}\right.$ and $\left.\tilde{X}_{2}>t x_{2}\right)$ is a regularly varying function with index $-1 / \eta$. Such an extra assumption is closely related to the second-order condition introduced in de Haan and Resnick (1993).

In multivariate EVT, the second-order condition characterizes the speed of convergence in (1.1) as follows: there exist a nonconstant function $\psi\left(x_{1}, x_{2}\right)$ and a positive function $A(t) \rightarrow$ 0 as $t \rightarrow \infty$ such that

$$
\begin{aligned}
\lim _{t \rightarrow \infty} & \frac{t \mathrm{P}\left(\left(X_{1}-b_{1}(t)\right) / a_{1}(t)>x_{1} \text { or }\left(X_{2}-b_{2}(t)\right) / a_{2}(t)>x_{2}\right)+\log G\left(x_{1}, x_{2}\right)}{A(t)} \\
\quad & =\psi\left(x_{1}, x_{2}\right)<\infty
\end{aligned}
$$

holds locally uniformly for $0<x_{1}, x_{2} \leq \infty$. This is a generalization of the second-order condition in the univariate case; see de Haan and Stadtmüller (1996). The second-order condition implies that $A(t)$ is a regularly varying function with index $\rho \leq 0$. Similarly to the first-order case, the second-order condition (1.6) implies that each marginal distribution $F_{i}$ satisfies the univariate second-order condition and, jointly, the dependence structure satisfies

$$
\lim _{t \rightarrow \infty} \frac{t\left(1-\mathrm{P}\left(\tilde{X}_{1} \leq t x_{1}, \tilde{X}_{2} \leq t x_{2}\right)\right)+\log G_{0}\left(x_{1}, x_{2}\right)}{A(t)}=\psi_{0}\left(x_{1}, x_{2}\right),
$$

where $\psi_{0}\left(x_{1}, x_{2}\right)=\psi\left(\left(x_{1}^{\gamma_{1}}-1\right) / \gamma_{1},\left(x_{2}^{\gamma_{2}}-1\right) / \gamma_{2}\right)$. With the notation of the exponent measure $v$, we have

$$
\lim _{t \rightarrow \infty} \frac{t \mathrm{P}\left(\tilde{X}_{1}>t x_{1}, \tilde{X}_{2}>t x_{2}\right)-v\left\{(u, v): u>x_{1} \text { and } v>x_{2}\right\}}{A(t)} \text { exists. }
$$

In the asymptotic independence case, this is simplified to

$$
\lim _{t \rightarrow \infty} \frac{\mathrm{P}\left(\tilde{X}_{1}>t x_{1}, \tilde{X}_{2}>t x_{2}\right)}{Q(t)} \text { exists and is positive, }
$$

where $Q(t):=A(t) / t$ is a positive regularly varying function with index $\rho-1 \leq-1$. By defining $\eta=1 /(1-\rho)$, the regularly varying index of $Q(t)$ is then $-1 / \eta$ and $\eta \leq 1$. In the case when $\eta<1$, we get the same setup as the Ledford and Tawn model. In the case when there is no asymptotic independence, $\eta=1$.

We say that there is extreme residual dependence if $\left(X_{1}, X_{2}\right)$ belongs to the domain of attraction of a bivariate extreme value distribution, they are asymptotic independent, and condition (1.7) holds. The name residual dependence reflects the fact that, after eliminating 
the basic independence, there is still notable dependence for the residual part. The parameter $\eta$ is called the extreme residual coefficient. Note that the $\eta$ corresponding to the case where the initial components $X_{1}$ and $X_{2}$ are independent is $\frac{1}{2}$. Only in the case $\eta>\frac{1}{2}$, the 'extremes' of $\left(X_{1}, X_{2}\right)$ are positively associated. In other words, the probability that one component is 'extreme' conditional on the other component being 'extreme' is higher than the corresponding unconditional probability. If $\eta<\frac{1}{2}$, the 'extremes' of the two dimensions turn out to be negatively associated, which is not regular for modeling joint tail events. Thus, we focus on the former case. An example is the bivariate normal distribution with correlation coefficient $r$ less than 1 . In this case $\eta=(1+r) / 2$. Moreover, $\eta<\frac{1}{2}$ corresponds to $r<0$, i.e. a negative correlation case. For more discussion, see also Remark 2.3 below.

There are quite a few papers on the 'Ledford and Tawn model' in $\mathbb{R}^{2}$. For instance, extreme residual dependence has been discussed under the name 'hidden regular variation' in Resnick (2002), Maulik and Resnick (2004), and Heffernan and Resnick (2005). Draisma et al. (2004) studied the estimation of the extreme residual coefficient. For application, Poon et al. (2004) applied both extreme value dependence and extreme residual dependence models in modeling financial returns from major stock indices. By estimating the extreme residual coefficient, they identified asymptotic dependence and independence among different pairs of stock indices.

For the characterization of extreme residual dependence, Ramos and Ledford (2009) considered bivariate regular variation and gave a characterization of extreme residual dependence in the two-dimensional case, which relies on a spectral measure restricted by a normalization condition. Due to the restriction, in application, it is not straightforward to verify the normalization condition, how to simulate random vectors exhibiting extreme residual dependence, and how to construct examples on extreme residual dependence from their characterization. Furthermore, although it is stated that the characterization in Ramos and Ledford (2009) can be extended to higher-dimensional cases, the connection with higher-dimensional EVT is not obvious.

We develop a theory characterizing the extreme residual dependence analogous to the traditional bivariate EVT as sketched in relations (1.3)-(1.5) above. Moreover, we generalize the Ledford and Tawn assumption into higher-dimensional Euclidean spaces as well as in the context of stochastic processes, i.e. the infinite-dimensional case. The generalization is not trivial in the following sense. In $\mathbb{R}^{2}$, the Ledford and Tawn assumption on extreme residual dependence implies a unique asymptotic independence structure on $v$ or $H: v$ must concentrate on the coordinate axes while $H$ must concentrate on the points $\{0\}$ and $\{1\}$. However, in the higher-dimensional case and in the stochastic process context, an analog of the Ledford and Tawn model does not correspond to a unique extreme value dependence structure exhibiting asymptotic independence. Instead, a variety of potential extreme value dependence structures may occur: asymptotic dependence may exist for some subsets of the components of the random vector but not for all components jointly. We will derive a full characterization of the extreme residual dependence that accommodates all potential dependence structures in all subdimensional marginals.

\section{Characterization of extreme residual dependence in $\mathbb{R}^{2}$}

We start with condition (1.7). It is equivalent to

$$
\lim _{t \rightarrow \infty} \frac{\mathrm{P}\left(\tilde{X}_{1}^{1 / \eta}>t x_{1} \text { and } \tilde{X}_{2}^{1 / \eta}>t x_{2}\right)}{Q\left(t^{\eta}\right)} \text { exists and is positive }
$$

for all $x_{1}, x_{2}>0$. Since $Q(t)$ is a regularly varying function with index $-1 / \eta, Q\left(t^{\eta}\right)$ is a regularly varying function with index -1 . 
Similarly to the two-dimensional EVT, there exists a measure $v^{*}$ on $(0,+\infty)^{2}$, finite on all sets $\left\{(u, v): u>x_{1}\right.$ and $\left.v>x_{2}\right\}$ for $x_{1}, x_{2}>0$, such that

$$
\lim _{t \rightarrow \infty} \frac{\mathrm{P}\left(\tilde{X}_{1}^{1 / \eta}>t x_{1} \text { and } \tilde{X}_{2}^{1 / \eta}>t x_{2}\right)}{Q\left(t^{\eta}\right)}=v^{*}\left\{(u, v): u>x_{1} \text { and } v>x_{2}\right\} .
$$

Clearly, for $a>0$ and a Borel set $B \subset(0,+\infty)^{2}$ that has a positive distance from both axes, i.e. $\inf _{\left(x_{1}, x_{2}\right) \in B} x_{1} \wedge x_{2}>0$, we have

$$
v^{*}(a B)=a^{-1} v^{*}(B) .
$$

Consider the following one-to-one transformation $(0,+\infty)^{2} \rightarrow(0, \infty) \times(0,1)$ :

$$
r(u, v)=\frac{1}{1 / u+1 / v}, \quad w(u, v)=\frac{r}{u} .
$$

Define, for constants $r>0$ and $w \in(0,1)$, the set

$$
B_{r, w}:=\left\{(u, v) \in(0,+\infty)^{2}: r(u, v)>r \text { and } 0<w(u, v) \leq w\right\} .
$$

Note that $v^{*}\left(B_{r, w}\right)<\infty$ for $r>0$ and $w \in(0,1)$, because $B_{r, w} \subset\{(u, v): u>r$ and $v>r\}$. Since $B_{r, w}=r B_{1, w}$, we have

$$
v^{*}\left(B_{r, w}\right)=r^{-1} v^{*}\left(B_{1, w}\right) .
$$

Set $H^{*}(w):=v^{*}\left(B_{1, w}\right)$ for $0<w<1$. Then $H^{*}$ is a finite measure on $(0,1)$. We show that all such measures occur. This is the main result in this section.

Theorem 2.1. Let $\left(X_{1}, X_{2}\right)$ be a random vector belonging to the domain of attraction of a twodimensional extreme value distribution. Suppose that $\left(X_{1}, X_{2}\right)$ are asymptotically independent with extreme residual dependence structure given in (2.1) and an extreme residual coefficient $\eta$ lying in $\left(\frac{1}{2}, 1\right)$. Then, there exists a finite measure $H^{*}$ on $(0,1)$ such that

$$
v^{*}\left\{(u, v): u>x_{1} \text { and } v>x_{2}\right\}=\int_{0}^{1} \frac{1}{x_{1} w} \wedge \frac{1}{x_{2}(1-w)} H^{*}(\mathrm{~d} w)
$$

for $x_{1}, x_{2}>0$. Conversely, for any finite measure $H^{*}$ on $(0,1)$, the right-hand side of (2.4) is positive and finite. Moreover, there exists a random vector $\left(X_{1}, X_{2}\right)$ exhibiting asymptotic independence in the two-dimensional EVT setup, and having extreme residual dependence structure given by (2.1) and (2.4).

Proof. Firstly, with the construction of $H^{*}$ above, we prove (2.4). Note that, for $r>0$ and $0<w<1$, the inverse of the transformation (2.3) is

$$
u(r, w)=\frac{r}{w}, \quad v(r, w)=\frac{r}{1-w} .
$$

The proof of (2.4) is then by calculation as follows:

$$
\begin{aligned}
v^{*}\left\{(u, v): u>x_{1} \text { and } v>x_{2}\right\} & =v^{*}\left\{(u, v): \frac{r(u, v)}{w(u, v)}>x_{1} \text { and } \frac{r(u, v)}{1-w(u, v)}>x_{2}\right\} \\
& =v^{*}\left\{(u, v): r(u, v)>x_{1} w(u, v) \vee x_{2}(1-w(u, v))\right\} \\
& =\int_{(0,1)} H^{*}(\mathrm{~d} w) \int_{r>x_{1} w \vee x_{2}(1-w)} \frac{1}{r^{2}} \mathrm{~d} r \\
& =\int_{(0,1)} \frac{1}{x_{1} w} \wedge \frac{1}{x_{2}(1-w)} H^{*}(\mathrm{~d} w) .
\end{aligned}
$$


Conversely, starting with any given finite measure $H^{*}$ on $(0,1)$, the measure $v^{*}$ defined via (2.4) satisfies

$$
\begin{aligned}
v^{*}\left\{(u, v): u>x_{1} \text { and } v>x_{2}\right\} & =\int_{(0,1)} \frac{1}{x_{1} w} \wedge \frac{1}{x_{2}(1-w)} H^{*}(\mathrm{~d} w) \\
& =\int_{(0,1)} \frac{1}{x_{1} w \vee x_{2}(1-w)} H^{*}(\mathrm{~d} w) \\
& \leq \int_{(0,1)} \frac{2}{x_{1} w+x_{2}(1-w)} H^{*}(\mathrm{~d} w) \\
& \leq \int_{(0,1)} \frac{2}{x_{1} \wedge x_{2}} H^{*}(\mathrm{~d} w) \\
& =\frac{2}{x_{1} \wedge x_{2}} H^{*}(0,1) \\
& <\infty
\end{aligned}
$$

for all $x_{1}, x_{2}>0$. Also, clearly, $v^{*}$ satisfies the homogeneity property (2.2). Next, we prove that any finite measure $H^{*}$ on $(0,1)$ may occur by constructing a suitable random vector $\left(U_{1}, U_{2}\right)$ verifying all the requirements.

Our construction is separated into two steps. In the first step we construct a random vector $\left(Z_{1}, Z_{2}\right)$ satisfying the residual dependence property.

Proposition 2.1. Given any finite measure $H^{*}$ on $(0,1)$, there exists a random vector $\left(Z_{1}, Z_{2}\right)$ such that, for any $x_{1}, x_{2}>0$,

$$
\begin{gathered}
\lim _{t \rightarrow \infty} t \mathrm{P}\left(Z_{1}>t x_{1} \text { and } Z_{2}>t x_{2}\right)=\int_{(0,1)} \frac{1}{x_{1} w} \wedge \frac{1}{x_{2}(1-w)} H^{*}(\mathrm{~d} w), \\
\lim _{t \rightarrow \infty} t^{\eta} \mathrm{P}\left(Z_{i}>t\right)=0 \quad \text { for } i=1,2
\end{gathered}
$$

Proof. Consider two independent random variables $R^{*}$ and $\Theta^{*}$ with distribution functions $\mathrm{P}\left(R^{*}>x\right)=d / x$ for $x \geq d$ and $\mathrm{P}\left(\Theta^{*}<w\right)=d^{-1} \int_{(0, w)} H^{*}(\mathrm{~d} w)$ for $0<w \leq 1$, where $d=H^{*}(0,1)$. Since $\frac{1}{2}<\bar{\eta}<1$, there exists a constant $\beta$ such that $1<\beta<1 / \eta$. Let

$$
Z_{1}=\frac{R^{*}}{\Theta^{*}} \wedge\left(R^{*}\right)^{\beta}, \quad Z_{2}=\frac{R^{*}}{1-\Theta^{*}} \wedge\left(R^{*}\right)^{\beta} .
$$

We first check relation (2.6) by calculation:

$$
\begin{aligned}
\lim _{t \rightarrow \infty} & t \mathrm{P}\left(Z_{1}>t x_{1} \text { and } Z_{2}>t x_{2}\right) \\
& =\lim _{t \rightarrow \infty} t \mathrm{P}\left(\frac{R^{*}}{\Theta^{*}}>t x_{1} \text { and } \frac{R^{*}}{1-\Theta^{*}}>t x_{2} \text { and }\left(R^{*}\right)^{\beta}>t\left(x_{1} \vee x_{2}\right)\right) \\
& =\lim _{t \rightarrow \infty} t \mathrm{E}_{\Theta^{*}} \frac{d}{t x_{1} \Theta^{*} \vee t x_{2}\left(1-\Theta^{*}\right) \vee t^{1 / \beta}\left(x_{1} \vee x_{2}\right)^{1 / \beta}} \\
& =\lim _{t \rightarrow \infty} \int_{(0,1)} \frac{1}{x_{1} w \vee x_{2}(1-w) \vee t^{1 / \beta-1}\left(x_{1} \vee x_{2}\right)^{1 / \beta}} H^{*}(\mathrm{~d} w) \\
& =\int_{(0,1)} \frac{1}{x_{1} w \vee x_{2}(1-w)} H^{*}(\mathrm{~d} w) .
\end{aligned}
$$


The final step comes from the Lebesgue dominated convergence theorem and the fact that the last integral is finite. We finish the proof of the proposition by verifying relation (2.7). Since $Z_{i} \leq\left(R^{*}\right)^{\beta}$,

$$
\begin{aligned}
\limsup _{t \rightarrow \infty} t^{\eta} \mathrm{P}\left(Z_{i}>t\right) & \leq \limsup _{t \rightarrow \infty} t^{\eta} \mathrm{P}\left(\left(R^{*}\right)^{\beta}>t\right) \\
& =\limsup _{t \rightarrow \infty} t^{\eta} \frac{d}{t^{1 / \beta}} \\
& =0 .
\end{aligned}
$$

Now we go back to the proof of Theorem 2.1. Let $\left(Z_{1}, Z_{2}\right)$ be the random vector constructed in the proof of Proposition 2.1. Let $\left(W_{1}, W_{2}\right)$ be independent random variables with distribution function $\mathrm{P}\left(W_{i}>x\right)=1 / x$ for $x \geq 1$. Let $\left(Z_{1}, Z_{2}\right)$ and $\left(W_{1}, W_{2}\right)$ be independent. In the second step we assemble them by $U_{i}:=W_{i} \vee Z_{i}^{\eta}, i=1,2$. We show that $\left(U_{1}, U_{2}\right)$ satisfies all the requirements in Theorem 2.1.

Firstly, we study the marginal distributions of $\left(U_{1}, U_{2}\right)$. Note that, for $i=1,2$ and $x>0$,

$$
\begin{aligned}
\lim _{t \rightarrow \infty} t \mathrm{P}\left(U_{i}>t x\right) & =\lim _{t \rightarrow \infty} t \mathrm{P}\left(W_{i} \vee Z_{i}^{\eta}>t x\right) \\
& =\lim _{t \rightarrow \infty} t \mathrm{P}\left(W_{i}>t x\right)+t \mathrm{P}\left(Z_{i}^{\eta}>t x\right)-t \mathrm{P}\left(W_{i}>t x\right) \mathrm{P}\left(Z_{i}^{\eta}>t x\right) \\
& =\frac{1}{x},
\end{aligned}
$$

because, by (2.7), only the first term contributes. By defining $F_{U_{i}}(x):=\mathrm{P}\left(U_{i} \leq x\right)$, we have $1 /\left(1-F_{U_{i}}(t)\right) \sim t$ as $t \rightarrow \infty$.

Next, we check the extremal dependence structure as in (2.1) and (2.4). From the construction we have

$$
\begin{array}{rl}
\lim _{t \rightarrow \infty} t & \mathrm{P}\left(U_{1}^{1 / \eta}>t x_{1} \text { and } U_{2}^{1 / \eta}>t x_{2}\right) \\
& =\lim _{t \rightarrow \infty} t \mathrm{P}\left(\left\{W_{1}^{1 / \eta}>t x_{1} \text { or } Z_{1}>t x_{1}\right\} \text { and }\left\{W_{2}^{1 / \eta}>t x_{2} \text { or } Z_{2}>t x_{2}\right\}\right) \\
& =\lim _{t \rightarrow \infty} t \mathrm{P}\left(\left(A_{1} \cup B_{1}\right) \cap\left(A_{2} \cup B_{2}\right)\right),
\end{array}
$$

where the sets are defined as $A_{i}:=\left\{W_{i}^{1 / \eta}>t x_{i}\right\}$ and $B_{i}:=\left\{Z_{i}>t x_{i}\right\}$. From the expansion

$$
\left(A_{1} \cup B_{1}\right) \cap\left(A_{2} \cup B_{2}\right)=\left(A_{1} A_{2}\right) \cup\left(B_{1} A_{2}\right) \cup\left(A_{1} B_{2}\right) \cup\left(B_{1} B_{2}\right),
$$

we obtain the lower and upper bounds for $P\left(\left(A_{1} \cup B_{1}\right) \cap\left(A_{2} \cup B_{2}\right)\right)$ as

$$
\mathrm{P}\left(B_{1} B_{2}\right) \leq \mathrm{P}\left(\left(A_{1} \cup B_{1}\right) \cap\left(A_{2} \cup B_{2}\right)\right) \leq \mathrm{P}\left(B_{1} B_{2}\right)+\mathrm{P}\left(B_{1} A_{2}\right)+\mathrm{P}\left(A_{1} B_{2}\right)+\mathrm{P}\left(A_{1} A_{2}\right) .
$$

Proposition 2.1 shows that, as $t \rightarrow \infty, t \mathrm{P}\left(B_{1} B_{2}\right)$ converges as in (2.6). To prove that $t \mathrm{P}\left(\left(A_{1} \cup\right.\right.$ $\left.\left.B_{1}\right) \cap\left(A_{2} \cup B_{2}\right)\right)$ converges to the same limit, we need to only verify that, as $t \rightarrow \infty, t \mathrm{P}\left(B_{1} A_{2}\right)$, $t \mathrm{P}\left(A_{1} B_{2}\right)$, and $t \mathrm{P}\left(A_{1} A_{2}\right)$ converge to 0 . Since the random vectors $\left(W_{1}, W_{2}\right)$ and $\left(Z_{1}, Z_{2}\right)$ are independent, considering (2.7) and the distribution function of $W_{i}$, we obtain

$$
\begin{aligned}
\lim _{t \rightarrow \infty} t \mathrm{P}\left(B_{1} A_{2}\right) & =\lim _{t \rightarrow \infty} t \mathrm{P}\left(Z_{1}>t x_{1}\right) \mathrm{P}\left(W_{2}>t^{\eta} x_{2}^{\eta}\right) \\
& =\lim _{t \rightarrow \infty} t \cdot o\left(t^{-\eta}\right) O\left(t^{-\eta}\right) \\
& =\lim _{t \rightarrow \infty} t^{1-2 \eta} o(1) \\
& =0
\end{aligned}
$$


due to $\frac{1}{2}<\eta<1$. Similarly, we obtain $\lim _{t \rightarrow \infty} t \mathrm{P}\left(A_{1} B_{2}\right)=0$. Furthermore, since $W_{1}$ and $W_{2}$ are independent, we obtain

$$
\lim _{t \rightarrow \infty} t \mathrm{P}\left(A_{1} A_{2}\right)=\lim _{t \rightarrow \infty} t \cdot O\left(t^{-\eta}\right) O\left(t^{-\eta}\right)=\lim _{t \rightarrow \infty} t^{1-2 \eta} O(1)=0
$$

Therefore, we have proved that (cf. (2.6))

$$
\begin{aligned}
\lim _{t \rightarrow \infty} t \mathrm{P}\left(U_{1}^{1 / \eta}>t x_{1} \text { and } U_{2}^{1 / \eta}>t x_{2}\right) & =\lim _{t \rightarrow \infty} t \mathrm{P}\left(Z_{1}>t x_{1} \text { and } Z_{2}>t x_{2}\right) \\
& =\int_{(0,1)} \frac{1}{x_{1} w} \wedge \frac{1}{x_{2}(1-w)} H^{*}(\mathrm{~d} w) .
\end{aligned}
$$

Together with the fact that $1 /\left(1-F_{U_{i}}(t)\right) \sim t$ as $t \rightarrow \infty$, relation (2.10) is equivalent to the extreme residual dependence condition (1.7).

In the last step, we check that $\left(U_{1}, U_{2}\right)$ belongs to the domain of attraction of a twodimensional extreme value distribution with asymptotic independence. Write

$$
\begin{aligned}
t \mathrm{P}\left(U_{1}>t x_{1} \text { or } U_{2}>t x_{2}\right)= & t \mathrm{P}\left(U_{1}>t x_{1}\right)+t \mathrm{P}\left(U_{2}>t x_{2}\right) \\
& -t \mathrm{P}\left(U_{1}>t x_{1} \text { and } U_{2}>t x_{2}\right) .
\end{aligned}
$$

From (2.9) and (2.10), the extreme value dependence structure is obvious.

Remark 2.1. Combining (2.1) and (2.4), we obtain

$$
\lim _{t \rightarrow \infty} \frac{\mathrm{P}\left(\tilde{X}_{1}>t x_{1}, \tilde{X}_{2}>t x_{2}\right)}{Q(t)}=\int_{0}^{1} \frac{1}{x_{1}^{1 / \eta} w} \wedge \frac{1}{x_{2}^{1 / \eta}(1-w)} H^{*}(\mathrm{~d} w)
$$

for all $x_{1}, x_{2}>0$, which gives the limit in (1.7). The limit is a combination of the extreme residual coefficient $\eta$ and the measure $H^{*}$. They can be independently chosen. It is different from the characterization in Ramos and Ledford (2009), which has a side condition on the two components.

Remark 2.2. Note that $v^{*}$ has the same homogeneity property as the exponent measure $v$; however, a $v^{*}$-measure is defined on Borel sets $B$ such that $\inf _{\left(x_{1}, x_{2}\right) \in B} x_{1} \wedge x_{2}>0$, while a $\nu$-measure is defined on Borel sets $B$ such that $\inf _{\left(x_{1}, x_{2}\right) \in B} x_{1} \vee x_{2}>0$. Hence, any exponent measure $v$ can act as a $v^{*}$-measure, but not vice versa. A $v^{*}$-measure can be extended to an exponent measure if and only if $v^{*}\left\{(u, v): u>x_{1}\right\}$ and $v^{*}\left\{(u, v): v>x_{2}\right\}$ are finite for all $x_{1}, x_{2}>0$. From (2.4), this is equivalent to the fact that $\int_{0}^{1}(1 / w) H^{*}(\mathrm{~d} w)$ and $\int_{0}^{1} 1 /(1-w) H^{*}(\mathrm{~d} w)$ are finite. In that case, we say that $H^{*}$ is of finite type. Otherwise, $H^{*}$ is called of infinite type.

Remark 2.3. Although the original Ledford and Tawn model only requires $0<\eta<1$, we consider $\frac{1}{2}<\eta<1$. The condition is crucial for the proof. On the other hand, the case in which $\eta>\frac{1}{2}$ is usually the one of interest in applications.

Remark 2.4. Theorem 2.1 gives the theoretical characterization for extreme residual dependence in the two-dimensional situation. In practice, the constructive proof gives a method for simulating such a random vector when the measure $H^{*}$ and the extreme residual coefficient $\eta$ are known. 


\section{Examples on extreme residual dependence in $\mathbb{R}^{2}$}

We consider a few examples to demonstrate the application of the characterization.

Example 3.1. Let $H^{*}$ be concentrated on $\frac{1}{2}$ with measure 1 . This is referred to as the $H_{1}$ measure.

With the $H_{1}$-measure, we obtain

$$
\int_{0}^{1} \frac{1}{x_{1} w \vee x_{2}(1-w)} H_{1}(\mathrm{~d} w)=2\left(\frac{1}{x_{1}} \wedge \frac{1}{x_{2}}\right) .
$$

Hence, inequality (2.5) turns out to be an equality in this case. To construct a random vector with such an extreme residual dependence structure, following the proof of Theorem 2.1, we may start by constructing $\left(Z_{1}, Z_{2}\right)$ as in (2.8). Note that, with the $H_{1}$-measure, $\Theta^{*}=\frac{1}{2}$ is a constant. We obtain $Z_{1}=Z_{2}=2 R^{*} \wedge\left(R^{*}\right)^{\beta}=$ : $Z$. Hence, with $\left(W_{1}, W_{2}\right)$ given as in the proof, by defining $U_{i}=W_{i} \vee Z^{\eta}$ for $i=1,2,\left(U_{1}, U_{2}\right)$ exhibits an extreme residual dependence structure characterized by the $H_{1}$-measure. Intuitively, this can be viewed as attaching a common factor $Z^{\eta}$ on the asymptotically independent random vector $\left(W_{1}, W_{2}\right)$. It is an analog of the completely tail-dependent case as in bivariate EVT, i.e. the extreme residual part exhibits the strongest dependency.

Example 3.2. Let $H^{*}$ be the uniform probability distribution on $(0,1)$. We define $H_{2}(w)=w$.

With the uniform distribution, $H_{2}(\mathrm{~d} w)=\mathrm{d} w$, we obtain

$$
\begin{aligned}
\int_{0}^{1} \frac{1}{x_{1} w \vee x_{2}(1-w)} H_{2}(\mathrm{~d} w) & =\left(\int_{0}^{x_{2} /\left(x_{1}+x_{2}\right)}+\int_{x_{2} /\left(x_{1}+x_{2}\right)}^{1}\right) \frac{1}{x_{1} w \vee x_{2}(1-w)} \mathrm{d} w \\
& =\frac{1}{x_{2}} \log \frac{x_{1}+x_{2}}{x_{1}}+\frac{1}{x_{1}} \log \frac{x_{1}+x_{2}}{x_{2}} .
\end{aligned}
$$

In Example 3.1, we have

$$
\int_{0}^{1} \frac{1}{w} H_{1}(\mathrm{~d} w)=\int_{0}^{1} \frac{1}{1-w} H_{1}(\mathrm{~d} w)=2<\infty .
$$

In Example 3.2, we have

$$
\int_{0}^{1} \frac{1}{w} H_{2}(\mathrm{~d} w)=\int_{0}^{1} \frac{1}{1-w} H_{2}(\mathrm{~d} w)=+\infty .
$$

Hence, $H_{1}$ is of finite-type measure, while $H_{2}$ is of infinite-type measure.

The last example is an asymmetric $H^{*}$-measure based on the beta distribution.

Example 3.3. Consider a measure $H_{3}^{(\alpha, \beta, c)}$ defined as

$$
H_{3}^{(\alpha, \beta, c)}(\mathrm{d} w)=c w^{\alpha-1}(1-w)^{\beta-1} \mathrm{~d} w,
$$

where $0<\alpha, \beta \leq+\infty$, and $c>0$ is an arbitrary positive constant.

By choosing the normalization constant $c=c(\alpha, \beta)$ properly, $H_{3}^{(\alpha, \beta, c(\alpha, \beta))}$ turns out to be a probability measure. It is then the beta distribution with parameters $\alpha, \beta$. It is clear that, when $\alpha \neq \beta, H_{3}^{(\alpha, \beta, c)}$ is an asymmetric measure on $(0,1)$. 
The values of $\alpha$ and $\beta$ show how much the $H_{3}^{(\alpha, \beta, c)}$ measure concentrates on the central part of $(0,1)$. Obviously, the uniform distribution in Example 3.2 corresponds to $\alpha=\beta=1$ and $c=1$. When normalizing the $H_{3}^{(\alpha, \beta, c)}$-measure to a probability measure, and taking $\alpha=\beta \rightarrow+\infty$, the limit is the $H_{1}$-measure in Example 3.1. Hence, this example covers the above two.

Moreover, it can be verified that $\int_{0}^{1}(1 / w) H_{3}^{(\alpha, \beta, c)}(\mathrm{d} w)$ is finite if and only if $\alpha>1$, while $\int_{0}^{1}(1 /(1-w)) H_{3}^{(\alpha, \beta, c)}(\mathrm{d} w)$ is finite if and only if $\beta>1$. Hence, for $1<\alpha, \beta \leq+\infty, H_{3}^{(\alpha, \beta, c)}$ is of finite type, while, for $0<\alpha \leq 1$ or $0<\beta \leq 1, H_{3}^{(\alpha, \beta, c)}$ of infinite type. Note that it is possible to have one of the two integrals finite, while the other integral is infinite.

Next, we calculate the explicit extreme residual dependence structure for a few specific values of $\alpha, \beta$, and $c$.

Take $\alpha=\beta=\frac{1}{2}$ and $c=\frac{1}{2}$. From

$$
\int_{x_{1} /\left(x_{1}+x_{2}\right)}^{1} \frac{(w(1-w))^{-1 / 2}}{w} \mathrm{~d} w=\sqrt{\frac{x_{2}}{x_{1}}}
$$

we have

$$
\int_{0}^{1} \frac{1}{x_{1} w \vee x_{2}(1-w)} H_{3}^{(1 / 2,1 / 2,1 / 2)}(\mathrm{d} w)=\frac{1}{\sqrt{x_{1} x_{2}}} .
$$

This is the extreme residual dependence structure from the bivariate normal distribution. As shown in Draisma et al. (2004, Example 2.1), a bivariate normal distribution with mean 0, variance 1 , and correlation coefficient $-1<r<1$ has extreme residual coefficient $\eta=(1+r) / 2$, and the corresponding limit in $(2.1)$ is $\sqrt{1 / x_{1} x_{2}}$. Hence, the density of the $H^{*}$-measure for the bivariate normal distribution is $\mathrm{d} w / 2 \sqrt{w(1-w)}$.

Take $\alpha=2, \beta=1$, and $c=2$. From

$$
\int_{x_{2} /\left(x_{1}+x_{2}\right)}^{1} \mathrm{~d} w=\frac{x_{1}}{x_{1}+x_{2}} \quad \text { and } \quad \int_{0}^{x_{2} /\left(x_{1}+x_{2}\right)} \frac{w}{1-w} \mathrm{~d} w=-\frac{x_{2}}{x_{1}+x_{2}}-\log \frac{x_{1}}{x_{1}+x_{2}},
$$

we have

$$
\int_{0}^{1} \frac{1}{x_{1} w \vee x_{2}(1-w)} H_{3}^{(2,1,2)}(\mathrm{d} w)=\frac{2}{x_{2}} \log \frac{x_{1}+x_{2}}{x_{1}} .
$$

This is a $H^{*}$-measure of infinite type because $\left(2 / x_{2}\right) \log \left(\left(x_{1}+x_{2}\right) / x_{1}\right)$ goes to $+\infty$ as $x_{1}$ tends to 0 . However, when taking $x_{2} \rightarrow 0$, it converges to a finite limit $2 / x_{1}$. It is an example in which one of the two integrals is finite, while the other integral is infinite.

\section{Extreme residual dependence in $\mathbb{R}^{d}, d \geq 3$}

The Ledford and Tawn model on extreme residual dependence is originally defined in the two-dimensional case. We generalize the definition of asymptotic independence and extreme residual dependence to random vectors in higher-dimensional Euclidean space $\mathbb{R}^{d}, d \geq 3$.

In Section 4.1, we review the EVT in $\mathbb{R}^{d}, d \geq 3$, and generalize the definition of asymptotic independence in the bivariate case to asymptotic joint independence in $\mathbb{R}^{d}, d \geq 3$. We provide the necessary and sufficient condition on the extreme value dependence structure corresponding to asymptotic joint independence.

In Section 4.2, we give the definition of extreme residual dependence in $\mathbb{R}^{d}, d \geq 3$, and show the difference from the bivariate case. Roughly speaking, in the higher-dimensional case $(d \geq 3)$, the extreme residual dependence condition corresponds to asymptotic joint 
independence, but does not uniquely determine the subdimensional dependence structure. We introduce a few compatibility conditions on the extreme residual dependence structure of a highdimensional random vector and that of the subdimensional marginals. Under the compatibility conditions, it is possible to give a full characterization of extreme dependence in $\mathbb{R}^{d}, d \geq 3$.

\subsection{Extreme value dependence in $\mathbb{R}^{d}, d \geq 3$, and asymptotic joint independence}

Suppose that a random vector $\left(X_{1}, X_{2}, \ldots, X_{d}\right)$ has joint distribution function $F\left(x_{1}, x_{2}, \ldots\right.$, $\left.x_{d}\right)$. Let $F_{i}\left(x_{i}\right), i=1,2, \ldots, d$, be the marginal distribution functions of $F$. Suppose that all the $F_{i}$ are continuous distribution functions. The distribution function $F$ is in the domain of attraction of a $d$-dimensional extreme value distribution if and only if $F_{i}$ belongs to the one-dimensional domain of attraction, and by defining $\tilde{X}_{i}:=1 /\left(1-F_{i}\left(X_{i}\right)\right)$, there exists a positive measure $v$ such that

$$
\lim _{t \rightarrow \infty} t \mathrm{P}\left(\tilde{X}_{1}>t x_{1} \text { or } \ldots \text { or } \tilde{X}_{d}>t x_{d}\right)=v\left\{\left(t_{1}, \ldots, t_{d}\right): t_{1}>x_{1} \text { or } \ldots \text { or } t_{d}>x_{d}\right\} .
$$

Again, the measure $v$ is the exponent measure. It characterizes the limiting extreme value distribution.

For $d \geq 3$, the extreme dependence can appear in several forms. Let us concentrate on the case $d=3$. There are three levels of extreme value dependence.

1. The measure $v$ is concentrated on the three axes. Then

$$
\lim _{t \rightarrow \infty} t \mathrm{P}\left(\tilde{X}_{i}>t x_{i} \text { and } \tilde{X}_{j}>t x_{j}\right)=0
$$

for all $i \neq j$ and $x_{i}, x_{j}>0$, and, hence, for all $x_{1}, x_{2}, x_{3}>0$,

$$
\lim _{t \rightarrow \infty} t \mathrm{P}\left(\tilde{X}_{1}>t x_{1} \text { and } \tilde{X}_{2}>t x_{2} \text { and } \tilde{X}_{3}>t x_{3}\right)=0 .
$$

2. The measure $v$ is concentrated on the planes $\left\{\left(t_{1}, t_{2}, t_{3}\right): t_{i}=0\right\}$ for $i=1,2,3$, but not only on the axes. Then one or more pairs $\left(X_{i}, X_{j}\right)$ are not asymptotically independent, but (4.2) still holds. For the other pairs, the theory of Section 2 applies.

3. The measure $v$ assigns some positive measure on the open area

$$
\left\{\left(t_{1}, t_{2}, t_{3}\right): t_{1}>0, t_{2}>0, t_{3}>0\right\} .
$$

Then (4.2) does not hold. All pairs $\left(X_{i}, X_{j}\right)$ are asymptotically dependent.

Hence, (4.2) should be considered in defining asymptotic independence in $\mathbb{R}^{3}$. For the general case $d \geq 3$, we say that the random vector $\left(X_{1}, \ldots, X_{d}\right)$ is asymptotically jointly independent if and only if

$$
\lim _{t \rightarrow \infty} t \mathrm{P}\left(\tilde{X}_{1}>t x_{1} \text { and } \ldots \text { and } \tilde{X}_{d}>t x_{d}\right)=0
$$

or, equivalently,

$$
v\left\{\left(t_{1}, \ldots, t_{d}\right): t_{1}>x_{1} \text { and } \ldots \text { and } t_{d}>x_{d}\right\}=0
$$

for any $x_{1}, x_{2}, \ldots, x_{d}>0$. This is the same as

$$
\nu\left\{\left(t_{1}, t_{2}, \ldots, t_{d}\right): t_{1}>0 \text { and } \ldots \text { and } t_{d}>0\right\}=0 .
$$




\subsection{Extreme residual dependence in $\mathbb{R}^{d}, d \geq 3$}

In the setup of Section 4.1, if the asymptotically jointly independent case, i.e. (4.3), holds, as an analog of the bivariate case, we can define extreme residual dependence in $\mathbb{R}^{d}$ as follows. If in that case there exists a regularly varying function $Q(t)$ with index $-1 / \eta$ such that

$$
\lim _{t \rightarrow \infty} \frac{\mathrm{P}\left(\tilde{X}_{1}>t x_{1} \text { and } \ldots \text { and } \tilde{X}_{d}>t x_{d}\right)}{Q(t)} \text { exists and is positive }
$$

for any $x_{1}, x_{2}, \ldots, x_{d}>0$, we say that there is extreme residual dependence with extreme residual coefficient $\eta$.

As shown in the proof of Theorem 2.1 in the two-dimensional case, the extreme residual dependence condition (2.1) automatically implies the EVT setup for $\tilde{X}_{i}, i=1,2$, with a unique spectral measure $v$ : $v$ must concentrate its measure on two axes, i.e. the extreme value dependence structure under asymptotic independence is unique. However, as discussed in the cases 1 and 2 in Section 4.1, in the case $d=3$, a given extreme residual dependence structure may not determine the three-dimensional extreme value dependence structure. In the next example, we further show that the two-dimensional marginals can be either extreme value dependent or extreme residual dependent, and the dependence structure in either case can be independent of the three-dimensional extreme residual dependence structure.

Let $\left(E_{1}, E_{2}\right)$ and $\left(E_{3}, E_{4}\right)$ be asymptotically dependent random vectors with standard Pareto marginals and exponent measures $v_{1}$ and $v_{2}$, i.e. $\mathrm{P}\left(E_{i}>x\right)=1 / x$ for $x>1$ and $i=1,2,3,4$, and

$$
\begin{aligned}
& \lim _{t \rightarrow \infty} t \mathrm{P}\left(E_{1}>t x_{1} \text { and } E_{2}>t x_{2}\right)=v_{1}\left\{(u, v): u>x_{1} \text { and } v>x_{2}\right\}>0, \\
& \lim _{t \rightarrow \infty} t \mathrm{P}\left(E_{3}>t x_{1} \text { and } E_{4}>t x_{2}\right)=v_{2}\left\{(u, v): u>x_{1} \text { and } v>x_{2}\right\}>0,
\end{aligned}
$$

for all $x_{1}, x_{2}>0$. Suppose that the two vectors are independent. Let $E_{5}$ and $E_{6}$ be two independent standard Pareto distributed random variables. Moreover, they are independent from the two aforementioned random vectors. We construct six factors $L_{1}, \ldots, L_{6}$ as follows:

$$
L_{i}=E_{i}, \quad \text { for } i=1,2, \quad L_{i}=E_{i}^{2 / 3} \quad \text { for } i=3,4, \quad L_{5}=E_{5}, \quad L_{6}=E_{6}^{2 / 5} .
$$

Then, we construct a three-dimensional random vector $\left(V_{1}, V_{2}, V_{3}\right)$ from the six factors as

$$
V_{1}=\max \left(L_{1}, L_{3}, L_{6}\right), \quad V_{2}=\max \left(L_{2}, L_{6}\right), \quad V_{3}=\max \left(L_{5}, L_{4}, L_{6}\right) .
$$

It is not difficult to verify that the tail parts of the one-dimensional marginal distributions of $\left(V_{1}, V_{2}, V_{3}\right)$ are dominated by the factors $L_{1}, L_{2}$, and $L_{5}$, i.e. $1-F_{V_{i}}(t):=\mathrm{P}\left(V_{i}>t\right) \sim 1 / t$ as $t \rightarrow \infty$ for $i=1,2,3$. Thus, $1 /\left(1-F_{V_{i}}(t)\right) \sim t$. When evaluating the joint dependence, it is sufficient to consider $V_{i}$ instead of $\tilde{V}_{i}=1 /\left(1-F_{V_{i}}\left(V_{i}\right)\right)$. We start with the extreme value dependence structure as follows:

$$
\begin{array}{rl}
\lim _{t \rightarrow \infty} t & \mathrm{P}\left(V_{1}>t x_{1} \text { or } V_{2}>t x_{2} \text { or } V_{3}>t x_{3}\right) \\
& =\lim _{t \rightarrow \infty} t \mathrm{P}\left(L_{1}>t x_{1} \text { or } L_{2}>t x_{2} \text { or } L_{5}>t x_{3}\right) \\
& =\lim _{t \rightarrow \infty} t \mathrm{P}\left(E_{1}>t x_{1} \text { or } E_{2}>t x_{2}\right)+t \mathrm{P}\left(E_{5}>t x_{3}\right) \\
& =v_{1}\left\{(u, v): u>x_{1} \text { or } v>x_{2}\right\}+\frac{1}{x_{3}} .
\end{array}
$$


Hence, the extreme value dependence structure of $\left(V_{1}, V_{2}, V_{3}\right)$ is the same as that of $\left(L_{1}, L_{2}, L_{5}\right)$, and depends on the choice of $v_{1}$. With respect to the two-dimensional marginals, $\left(V_{1}, V_{2}\right)$ are tail dependent, with an extreme value dependence structure given by $v_{1}$, while $\left(V_{1}, V_{3}\right)$ and $\left(V_{2}, V_{3}\right)$ are tail independent.

Next, we have the following statements.

1. $\left(V_{1}, V_{3}\right)$ exhibits extreme residual dependence with an extreme residual coefficient $\eta_{\{1,3\}}=\frac{2}{3}$, while the extreme residual dependence structure is associated to $v_{2}$.

2. $\left(V_{2}, V_{3}\right)$ exhibits extreme residual dependence with an extreme residual coefficient $\eta_{\{2,3\}}=\frac{3}{5}$, while the extreme residual dependence structure is given by

$$
\lim _{t \rightarrow \infty} t^{5 / 3} \mathrm{P}\left(V_{2}>t x_{2}, V_{3}>t x_{3}\right)=\frac{1}{\max \left(x_{2}, x_{3}\right)^{5 / 3}} .
$$

3. $\left(V_{1}, V_{2}, V_{3}\right)$ exhibits extreme residual dependence with an extreme residual coefficient $\eta_{\{1,2,3\}}=\frac{3}{5}$, while the extreme residual dependence structure is given by

$$
\lim _{t \rightarrow \infty} t^{5 / 3} \mathrm{P}\left(V_{1}>t x_{1}, V_{2}>t x_{2}, V_{3}>t x_{3}\right)=\frac{1}{\max \left(x_{1}, x_{2}, x_{3}\right)^{5 / 3}} .
$$

The statements can be proved by set manipulations. Roughly speaking, the extreme residual dependence structures of $\left(V_{1}, V_{3}\right),\left(V_{2}, V_{3}\right)$, and $\left(V_{1}, V_{2}, V_{3}\right)$ are driven by $\left(L_{3}, L_{4}\right),\left(L_{6}, L_{6}\right)$, and $\left(L_{6}, L_{6}, L_{6}\right)$, respectively.

From the three statements, we obtain the following facts for the constructed threedimensional random vector which exhibits extreme residual dependence. Firstly, since $v_{1}$ is free to choose, it implies that the three-dimensional extreme residual dependence structure does not determine the extreme value dependence structure of the random vector. Secondly, from the extreme value dependence structure of $\left(V_{1}, V_{2}\right)$, it is possible to have extreme value dependence for subdimensional marginals, but the extreme value dependence structure must be the projection of that of the three-dimensional random vector. Thirdly, from the extreme residual dependence in $\left(V_{1}, V_{3}\right)$ and $\left(V_{2}, V_{3}\right)$, it is possible to have extreme residual dependence with a higher or equal extreme residual coefficient for subdimensional marginals. On the one hand, when the subdimensional extreme residual coefficient is higher than that of the threedimensional random vector $\left(\eta_{\{1,3\}}>\eta_{\{1,2,3\}}\right)$, the corresponding extreme residual dependence structure is independent from that of the three-dimensional random vector. This is shown by the fact that $v_{2}$ is free to choose. On the other hand, when the subdimensional extreme residual coefficient is equal to that of the three-dimensional random vector $\left(\eta_{\{2,3\}}=\eta_{\{1,2,3\}}\right)$, the extreme residual dependence structure of $\left(V_{2}, V_{3}\right)$ must be a projection of that of the three-dimensional random vector.

Although having an extreme residual dependence structure for a high-dimensional random vector does not determine the subdimensional dependence structure, some compatibility conditions must be obeyed by the subdimensional dependence structure. We present the compatibility conditions under a general $d$-dimensional framework. The proofs are straightforward and thus omitted. Consider a $d$-dimensional random vector $\left(X_{1}, X_{2}, \ldots, X_{d}\right)$. By taking $\tilde{X}_{i}=1 /\left(1-F_{i}\left(X_{i}\right)\right)$, where $F_{i}$ is the marginal distribution of $X_{i}$, we get the random vector $\left(\tilde{X}_{1}, \tilde{X}_{2}, \ldots, \tilde{X}_{d}\right)$. Suppose that, for any subset of index $A \subset\{1,2, \ldots, d\}$, there exists a regularly varying function $Q_{A}(t)$ with index $-1 / \eta_{A}$ such that, for any $x_{i}>0, i=1,2, \ldots, d$, the limit

$$
\lim _{t \rightarrow \infty} \frac{\mathrm{P}\left(\bigcap_{i \in A}\left\{\tilde{X}_{i}>t x_{i}\right\}\right)}{Q_{A}(t)}
$$


exists and is positive. By normalizing (4.4) we have

$$
\lim _{t \rightarrow \infty} \frac{\mathrm{P}\left(\bigcap_{i \in A}\left\{\tilde{X}_{i}^{1 / \eta_{A}}>t x_{i}\right\}\right)}{Q_{A}\left(t^{\eta_{A}}\right)}=v_{A}^{*}\left\{\left(u_{i}, i \in A\right): u_{i}>x_{i} \text { for all } i \in A\right\},
$$

where $v_{A}^{*}$ is a finite measure on $(0,+\infty)^{|A|}$, homogeneous with degree -1 . Note that we do not distinguish extreme value dependence and extreme residual dependence. Instead, we regard the extreme value dependence as a special case when $\eta_{A}=1$.

Then we have the following conditions.

Condition 1: one-dimensional coefficient. For all sets $A$ such that $|A|=1$, where $|\cdot|$ denotes the number of elements in a set, $\eta_{A}=1$.

Condition 2: monotonicity. For any set $A \subset B, \eta_{A} \geq \eta_{B}$.

Condition 3: consistency. If $A \subset B$ and $\eta_{A}=\eta_{B}$, the measure $v_{A}^{*}$ is a projection of the measure $v_{B}^{*}$. Formally, for any Borel set $S \subset(0,+\infty)^{|A|}$, the inverse projection $\pi(S) \subset(0,+\infty)^{|B|}$ is defined as

$$
\pi(S):=\left\{\left(x_{i}\right)_{i \in B}:\left(x_{i}\right)_{i \in A} \in S,\left(x_{i}\right)_{i \in B / A} \in(0,+\infty)\right\} .
$$

The projection $v_{A}^{*}$ of $v_{B}^{*}$ is defined as follows: for any $T \subset(0,+\infty)^{|A|}, v_{A}^{*}(T)=$ $v_{B}^{*}(\pi(T))$.

Similarly to the two-dimensional case, when we confine ourselves to the case that the 'extremes' are positively associated, we have the following condition.

Condition 4: positive dependence. Suppose that $A=A_{1} \cup A_{2}$ is a nonempty partition of the set $A$, i.e. $A_{1} \cap A_{2}=\varnothing$ and $A_{j} \neq \varnothing$ for $j=1$, 2. Then $1 / \eta_{A}<1 / \eta_{A_{1}}+1 / \eta_{A_{2}}$.

Note that if the random vector $\left(X_{i}\right)_{i \in A_{1}}$ is independent from $\left(X_{i}\right)_{i \in A_{2}}, 1 / \eta_{A}=1 / \eta_{A_{1}}+1 / \eta_{A_{2}}$. Thus, the inequality ensures that the 'extremes' are positively associated. In the two-dimensional case, condition 4 turns out to be $\eta>\frac{1}{2}$. Conversely, condition 4 can be regarded as a generalization of the $\eta>\frac{1}{2}$ condition in the two-dimensional case.

With the compatibility conditions $1-4$, we have the full characterization of the extreme dependence structure for a high-dimensional random vector.

Theorem 4.1. Let $\left(X_{1}, X_{2}, \ldots, X_{d}\right)$ be a random vector belonging to the domain of attraction of a d-dimensional extreme value distribution, and let the extreme value dependence be characterized by an exponent measure $v$ as in (4.1). Suppose that, for any index set $A \subset\{1,2, \ldots, d\}$, we have the extreme dependence structure of $\left(X_{i}\right)_{i \in A}$ characterized by $\eta_{A}$ and $v_{A}^{*}$. In the case $\eta_{A}=1$, it characterizes the extreme value dependence structure, while in the case $\eta_{A}>1$, it characterizes the extreme residual dependence structure. Then the characterization set $\left\{\left(\eta_{A}, v_{A}^{*}\right): A \subset\{1,2, \ldots, d\}\right\}$ must satisfy the compatibility conditions $1-3$, while each $v_{A}^{*}$ measure must be finite on all Borel sets in $(0,+\infty)^{|A|}$ and homogeneous with degree -1 .

Conversely, given any characterization set $\left\{\left(\eta_{A}, v_{A}^{*}\right): A \subset\{1,2, \ldots, d\}\right\}$ satisfying the compatibility conditions $1-4$, there exists a random vector $\left(X_{1}, X_{2}, \ldots, X_{d}\right)$ such that all subdimensional extreme dependence structures are characterized by corresponding coefficients and $v^{*}$-measures.

We skip the proof of Theorem 4.1 because it follows similar lines as the proof for the twodimensional case, while the constructed three-dimensional example gives a general idea of the constructive proof. 
Remark 4.1. From the compatibility conditions 1 and 4 , we obtain $\eta_{A}>1 /|A|$.

\section{Extreme residual dependence for stochastic processes}

We consider stochastic processes defined on the unit interval $[0,1]$. Let $\{X(s)\}_{s \in[0,1]}$ be a continuous sample path stochastic process, i.e. a random element in the space $C[0,1]$ equipped with the $L_{\infty}$ norm $|f|_{\infty}:=\sup _{s \in[0,1]} f(s)$. In this section we generalize the definition of asymptotic joint independence and extreme residual dependence to continuous sample path stochastic processes.

In Section 5.1 we review the EVT in $C[0,1]$ and study the concept of asymptotic joint independence in the infinite-dimensional case. In Section 5.2 we generalize the definition of extreme residual dependence into $C[0,1]$, and give its characterization.

\subsection{Extreme value dependence and asymptotic joint independence in $C[0,1]$}

Suppose that a continuous sample path stochastic process $\{X(s)\}_{s \in[0,1]}$ belongs to the domain of attraction of a max-stable process (for details, see de Haan and Ferreira (2006, Chapter 9)). Suppose that the marginal distribution function of $X(s), F_{s}(x)$, is a continuous distribution function. The domain of attraction condition is equivalent to the combination of two conditions: firstly, $F_{S}(x)$ belongs to the one-dimensional domain of attraction with extreme value index $\gamma(s)$, where $\gamma(s)$ is a continuous real-valued function on $[0,1]$; secondly, by defining

$$
\{\tilde{X}(s)\}_{s \in[0,1]}:=\left\{\frac{1}{1-F_{S}(X(s))}\right\}_{s \in[0,1]},
$$

$\{\tilde{X}(s)\}_{s \in[0,1]}$ is also a continuous sample path stochastic process belonging to the same domain of attraction. EVT in $C[0,1]$ shows that there must exist a finite measure $v$ on $\bar{C}^{+}[0,1]:=$ $\{f \in C[0,1]: f \geq 0\}$ such that, for all Borel sets $A \subset \bar{C}^{+}[0,1]$ with

$$
\inf \left\{|f|_{\infty}: f \in A\right\}>0
$$

and $v(\partial A)=0$,

$$
\lim _{t \rightarrow \infty} t \mathrm{P}(\tilde{X} \in t A)=v(A) .
$$

Moreover, $v$ is a homogeneous measure with degree -1 . A corresponding spectral measure $\rho$ is given in Giné et al. (1990) (cf. Theorem 9.4.1 of de Haan and Ferreira (2006)). For any $f \in \bar{C}^{+}[0,1]$ with $|f|_{\infty}>0$, we write $r:=|f|_{\infty}$ and

$$
f_{1}(s):=\frac{f(s)}{|f|_{\infty}} \in \bar{C}_{1}^{+}[0,1]:=\left\{f \in C[0,1]: f \geq 0,|f|_{\infty}=1\right\} .
$$

Then $f$ is decomposed into $\left(r, f_{1}\right) \in(0, \infty) \times \bar{C}_{1}^{+}[0,1]$. With such a decomposition, the exponent measure $v$ is decomposed into a product measure as follows. There exists a finite measure $\rho$ on $\bar{C}_{1}^{+}[0,1]$ with

$$
\int_{\bar{C}_{1}^{+}[0,1]} f_{1}(s) \mathrm{d} \rho\left(f_{1}\right)=1
$$

for any $s \in[0,1]$ such that

$$
v(A)=\iint_{r f_{1} \in A} \frac{\mathrm{d} r}{r^{2}} \mathrm{~d} \rho\left(f_{1}\right)
$$


Again, the measures $v$ and $\rho$ are called the exponent measure and the spectral measure, respectively. Either of them characterizes the extreme value dependence for the stochastic process $\{\tilde{X}(s)\}_{s \in[0,1]}$ and, hence, $\{X(s)\}_{s \in[0,1]}$.

We introduce the notation $f>g$ (or $f \geq g$ ) to indicate that two continuous functions $f(s)$ and $g(s)$ defined on $s \in[0,1]$ satisfy $f(s)>g(s)$ (or $f(s) \geq g(s)$ ) for all $s \in[0,1]$. Then, the extreme value dependence structure is equivalent to, for any $g \in C^{+}[0,1]:=\{f \in$ $C[0,1]: f>0\}$,

$$
\lim _{t \rightarrow \infty} t \mathrm{P}\left(\{\tilde{X} \leq t g\}^{\mathrm{c}}\right)=v\left(\left\{f \in \bar{C}^{+}[0,1]: f \leq g\right\}^{\mathrm{c}}\right)=\int_{\bar{C}_{1}^{+}[0,1]} \sup _{s \in[0,1]} \frac{f_{1}}{g} \mathrm{~d} \rho\left(f_{1}\right)>0 .
$$

Similarly to the definition of asymptotic joint independence in $\mathbb{R}^{d}, d \geq 3$, in (4.3), we say that the stochastic process $\{X(s)\}_{s \in[0,1]}$ is asymptotically jointly independent if and only if, for any $g \in C^{+}[0,1]$, the following relation holds:

$$
\lim _{t \rightarrow \infty} t \mathrm{P}(\{\tilde{X}>\operatorname{tg}\})=v\left(\left\{f \in \bar{C}^{+}[0,1]: f>g\right\}\right)=0,
$$

i.e. no information on the sets of the type $\left\{f \in \bar{C}^{+}[0,1]: f>g\right\}$ is available.

The following theorem provides the necessary and sufficient condition on the spectral measure which corresponds to asymptotic joint independence.

Theorem 5.1. Suppose that a continuous sample path stochastic process $\{X(s)\}_{s \in[0,1]}$ belongs to the domain of attraction of some max-stable process with spectral measure $\rho$. The process is asymptotically jointly independent if and only if its spectral measure $\rho$ satisfies

$$
\rho\left\{f_{1} \in \bar{C}_{1}^{+}[0,1]: \inf f_{1}(s)>0\right\}=0 .
$$

Proof. With the relation between the exponent measure $v$ and the spectral measure $\rho$ in (5.1), we obtain, for any $g \in C^{+}[0,1]$,

$$
\begin{aligned}
v\left(\left\{f \in \bar{C}^{+}[0,1]: f>g\right\}\right) & =\iint_{r f_{1}>g} \frac{\mathrm{d} r}{r^{2}} \mathrm{~d} \rho\left(f_{1}\right) \\
& =\int_{\bar{C}_{1}^{+}[0,1]}\left(\int_{r>\sup \left(g / f_{1}\right)} \frac{\mathrm{d} r}{r^{2}}\right) \mathrm{d} \rho\left(f_{1}\right) \\
& =\int_{\bar{C}_{1}^{+}[0,1]} \frac{1}{\sup \left(g / f_{1}\right)} \mathrm{d} \rho\left(f_{1}\right) \\
& =\int_{\bar{C}_{1}^{+}[0,1]} \inf \left(\frac{f_{1}}{g}\right) \mathrm{d} \rho\left(f_{1}\right) \\
& =\int_{\left\{f_{1} \in \bar{C}_{1}^{+}[0,1]: \inf f_{1}>0\right\}} \inf \left(\frac{f_{1}}{g}\right) \mathrm{d} \rho\left(f_{1}\right) .
\end{aligned}
$$

It is then obvious that condition (5.3) implies (5.2). Conversely, suppose that (5.2) holds for all $g \in C^{+}[0,1]$. Note that

$$
\inf \left(\frac{f_{1}}{g}\right) \geq \inf f_{1} \inf \left(\frac{1}{g}\right)=\inf f_{1} \frac{1}{\sup g}>0
$$

on the set $\left\{f_{1} \in \bar{C}_{1}^{+}[0,1]\right.$ : inf $\left.f_{1}>0\right\}$. Then, we obtain (5.3). 
Remark 5.1. It is not difficult to verify that condition (5.3) is equivalent to

$$
\int_{\bar{C}_{1}^{+}[0,1]} \inf f_{1}(s) \mathrm{d} \rho\left(f_{1}\right)=0 .
$$

Note (cf. Ferreira et al. (2009)) that

$$
\int_{\bar{C}_{1}^{+}[0,1]} \inf f_{1}(s) \mathrm{d} \rho\left(f_{1}\right)=\lim _{\gamma \downarrow-\infty} \theta_{\gamma},
$$

where

$$
\theta_{\gamma}:=\int_{\bar{C}_{1}^{+}[0,1]}\left(\int_{s \in[0,1]} f_{1}^{\gamma}(s) \mathrm{d} s\right)^{1 / \gamma} \mathrm{d} \rho\left(f_{1}\right)
$$

is the so-called areal coefficient in Coles and Tawn (1996).

\subsection{Extreme residual dependence in $C[0,1]$}

We generalize the definition of extreme residual dependence for stochastic processes. Since we have infinitely many combinations of subdimensions, it is not possible give a full characterization that addresses all subdimensional dependence. We focus on the characterization of the extreme residual dependence over the index set $[0,1]$.

Suppose that a continuous sample path stochastic process $\{X(s)\}_{s \in[0,1]}$ belonging to the domain of attraction of some max-stable process is asymptotically jointly independent, i.e. (5.2) holds. Analogous to (1.7), if there exists a regularly varying function $Q(t)$ with index $-1 / \eta$ for some $0<\eta<1$ such that

$$
\lim _{t \rightarrow \infty} \frac{\mathrm{P}(\{\tilde{X}>t g\})}{Q(t)} \text { exists and is positive }
$$

for any $g \in C^{+}[0,1]$, we say that there is extreme residual dependence with extreme residual coefficient $\eta$.

From the definition of extreme residual dependence in (5.4) we obtain

$$
\lim _{t \rightarrow \infty} \frac{\mathrm{P}\left(\left\{\tilde{X}^{1 / \eta}>t g\right\}\right)}{Q\left(t^{\eta}\right)} \text { exists and is positive. }
$$

Hence, there must exist a measure $v^{*}$ on $C^{+}[0,1]$, finite on sets of the type $A_{g}^{*}:=\{f \in$ $C[0,1]: f>g\}$ for any $g \in C^{+}[0,1]$, such that

$$
\lim _{t \rightarrow \infty} \frac{\mathrm{P}\left(\left\{\tilde{X}^{1 / \eta}>t g\right\}\right)}{Q\left(t^{\eta}\right)}=v^{*}\left(A_{g}^{*}\right) .
$$

For any $a>0$, it can be verified that

$$
v^{*}\left(A_{a g}^{*}\right)=a^{-1} v^{*}\left(A_{g}^{*}\right)
$$

Hence, we obtain

$$
v^{*}(a A)=a^{-1} v^{*}(A)
$$

for all $A$ in the $\sigma$-field generated by $\left\{A_{g}^{*}: g \in C^{+}[0,1]\right\}$. 
To obtain a spectral decomposition of $v^{*}$, we consider the following transformation. For any $f \in C^{+}[0,1]$, since $f$ is a continuous function on a compact interval, we have $m(f):=$ $\inf _{s \in[0,1]} f(s)>0$. Moreover, by defining $f_{2}(s):=m / f(s)$, we have $f_{2} \in C_{1}^{+}[0,1]:=\{f \in$ $\left.C^{+}[0,1]:|f|_{\infty}=1\right\}$. Thus, any $f \in C^{+}[0,1]$ is decomposed into $\left(m, f_{2}\right) \in(0,+\infty) \times$ $C_{1}^{+}[0,1]$. For any Borel set $B \subset C_{1}^{+}[0,1]$ and any $r>0$, we obtain

$$
v^{*}\left\{f: m(f)>r, f_{2} \in B\right\}=\frac{1}{r} v^{*}\left\{f: m(f)>1, f_{2} \in B\right\} .
$$

By defining $\Psi^{*}(B):=v^{*}\left\{f: m(f)>1, f_{2} \in B\right\}$, we decompose the measure $v^{*}$ into a product measure on $(0,+\infty) \times C_{1}^{+}[0,1]$. The measures $\nu^{*}$ and $\Psi^{*}$ are connected by

$$
v^{*}(A)=\iint_{m / f_{2} \in A} \frac{\mathrm{d} m}{m^{2}} \mathrm{~d} \Psi^{*}\left(f_{2}\right) .
$$

Since

$$
\Psi^{*}\left(C_{1}^{+}[0,1]\right)=v^{*}\{f: m(f)>1\}=v^{*}\{f: f>1\}<\infty,
$$

$\Psi^{*}$ is a finite measure on $C_{1}^{+}[0,1]$.

Conversely, taking any finite measure $\Psi^{*}$ on $C_{1}^{+}[0,1]$, we construct $v^{*}$ as above. Then,

$$
\begin{aligned}
v^{*}\left(A_{g}^{*}\right) & =v^{*}\{f \in C[0,1]: f>g\} \\
& =v^{*}\left\{f \in C[0,1]: \frac{m}{f_{2}}>g\right\} \\
& =v^{*}\left\{f \in C[0,1]: m>\sup \left(g f_{2}\right)\right\} \\
& =\int_{C_{1}^{+}[0,1]} \frac{1}{\sup \left(g f_{2}\right)} \mathrm{d} \Psi^{*}\left(f_{2}\right) \\
& \leq \int_{C_{1}^{+}[0,1]} \frac{1}{\sup f_{2} \cdot \inf g} \mathrm{~d} \Psi^{*}\left(f_{2}\right) \\
& =\frac{1}{\inf g} \Psi^{*}\left(C_{1}^{+}[0,1]\right) .
\end{aligned}
$$

Hence, for all continuous functions $g>0, v^{*}\left(A_{g}^{*}\right)<\infty$. Moreover, it is obvious that the constructed $v^{*}$-measure satisfies the homogeneity condition (5.5).

To summarize, we have shown that the extreme residual dependence condition can be characterized by either a measure $v^{*}$ on $C^{+}[0,1]$ or a measure $\Psi^{*}$ on $C_{1}^{+}[0,1]$. The former must be finite on all sets of the type $A_{g}^{*}$ and satisfying (5.5), while the latter must be a finite measure on $C_{1}^{+}[0,1]$.

As shown in Theorem 5.1, when the extreme residual dependence condition holds, the spectral measure $\rho$ must satisfy condition (5.3). The following theorem shows that all possible extreme value dependence structures and extreme residual dependence structures can occur.

Theorem 5.2. Consider a continuous sample path stochastic process $\{X(s)\}_{s \in[0,1]}$ in the domain of attraction of a max-stable process with spectral measure $\rho$. Suppose that $\{X(s)\}_{s \in[0,1]}$ is asymptotically jointly independent and exhibits extreme residual dependence characterized by an extreme residual coefficient $\eta$ in $\left(\frac{1}{2}, 1\right)$ and a measure $\Psi^{*}$. Then the spectral measure $\rho$ must satisfy (5.3), and the measure $\Psi^{*}$ is finite on $C_{1}^{+}[0,1]$.

Conversely, given a spectral measure $\rho$ satisfying (5.3), a finite measure $\Psi^{*}$ on $C_{1}^{+}[0,1]$, and any $\frac{1}{2}<\eta<1$, there exists a process $\{X(s)\}_{s \in[0,1]}$ belonging to the domain of attraction 
of a max-stable process with spectral measure $\rho$, exhibiting extreme residual dependence characterized by the given measure $\Psi^{*}$ and having an extreme residual coefficient $\eta$.

5.2.1. Proof of Theorem 5.2. The first part is proved by Theorem 5.1 and the construction of $\Psi^{*}$. It is only necessary to prove the inverse part. Similarly to the two-dimensional case, we give a constructive proof. The proof is staged in three steps.

Firstly, we construct a stochastic process $\{W(s)\}_{s \in[0,1]}$ with extreme value dependence structure characterized by $\rho$. Although this has been proved in Theorem 9.4.1 of de Haan and Ferreira (2006), we provide our own construction which is necessary for the proof later.

Define $c_{0}=\rho\left(\bar{C}_{1}^{+}[0,1]\right)<\infty$. Then $\rho / c_{0}$ is a probability distribution on $\bar{C}_{1}^{+}[0,1]$. Let $Q_{0}$ be a random element on $\bar{C}_{1}^{+}[0,1]$ following such a probability distribution. Let $M_{0}$ be a random variable independent from $Q_{0}$, with distribution function $\mathrm{P}\left(M_{0}>x\right)=c_{0} / x$ for all $x>c_{0}$. Then the constructed stochastic process $W$ is given as

$$
\{W(s)\}_{s \in[0,1]}:=\left\{M_{0} Q_{0}(s)\right\}_{s \in[0,1]} .
$$

It is not difficult to verify that the marginal distributions of the stochastic process $\{W(s)\}_{s \in[0,1]}$ follow

$$
\lim _{t \rightarrow \infty} t \mathrm{P}(\{W(s)>t x\})=\frac{1}{x} .
$$

Furthermore, for any $g \in C^{+}[0,1]$,

$$
\begin{aligned}
\lim _{t \rightarrow \infty} t \mathrm{P}\left(\{W \leq t g\}^{c}\right) & =\lim _{t \rightarrow \infty} t \mathrm{P}\left(\left\{M_{0}>t \inf _{s \in[0,1]} \frac{g}{Q_{0}}\right\}\right) \\
& =\mathrm{E} \frac{c_{0}}{\inf _{s \in[0,1]} g / Q_{0}} \\
& =\int_{\bar{C}_{1}^{+}[0,1]} \sup _{s \in[0,1]} \frac{f_{1}}{g} \mathrm{~d} \rho\left(f_{1}\right) .
\end{aligned}
$$

Together with the marginal property (5.7), the process $\{W(s)\}_{s \in[0,1]}$ is in the domain of attraction of a max-stable process with spectral measure $\rho$.

Secondly, we construct a stochastic process that accommodates the extreme residual dependence characterized by $\Psi^{*}$ and $\eta$ as in the following proposition.

Proposition 5.1. Given any finite measure $\Psi^{*}$ on $C_{1}^{+}[0,1]$ and $\frac{1}{2}<\eta<1$, there exists a continuous sample path stochastic process $\{Z(s)\}_{s \in[0,1]}$ such that, for any $g \in C^{+}[0,1]$,

$$
\begin{aligned}
& \lim _{t \rightarrow \infty} t \mathrm{P}(\{Z>t g\})=\int_{C_{1}^{+}[0,1]} \frac{1}{\sup \left(g f_{2}\right)} \mathrm{d} \Psi^{*}\left(f_{2}\right), \\
& \lim _{t \rightarrow \infty} t^{\eta} \mathrm{P}(Z(s)>t)=0 \text { for } s \in[0,1] .
\end{aligned}
$$

Proof. The proof is similar to the two-dimensional case. Define $c:=\Psi^{*}\left(C_{1}^{+}[0,1]\right)<\infty$. Then $\Psi^{*} / c$ is a probability distribution on $C_{1}^{+}[0,1]$. Consider a random element $Q$ in $C_{1}^{+}[0,1]$ following such a probability distribution. Let $M$ be a random variable independent from $Q$, with distribution function $\mathrm{P}(M>x)=c / x$ for all $x>c$. Moreover, since $\frac{1}{2}<\eta<1$, we take $\beta$ such that $1<\beta<1 / \eta$. Consider the stochastic process $\{Z(s)\}_{s \in[0,1]}$ given as

$$
\{Z(s)\}_{s \in[0,1]}:=\left\{\frac{M}{Q(s)} \wedge M^{\beta}\right\}_{s \in[0,1]} .
$$


It is clear that $\{Z(s)\}_{s \in[0,1]}$ is a continuous sample path process. We check condition (5.9) as follows. For any $g \in C^{+}[0,1]$,

$$
\begin{aligned}
\lim _{t \rightarrow \infty} t \mathrm{P}(\{Z>t g\}) & =\lim _{t \rightarrow \infty} t \mathrm{P}\left(\{M>t Q g\} \cap\left\{M^{\beta}>t \sup _{s \in[0,1]} g(s)\right\}\right) \\
& =\lim _{t \rightarrow \infty} t \mathrm{P}\left(\left\{M>(t \sup (Q g)) \vee\left(t^{1 / \beta}(\sup g)^{1 / \beta}\right)\right\}\right) \\
& =\lim _{t \rightarrow \infty} t \mathrm{E}_{Q} \frac{c}{(t \sup (Q g)) \vee\left(t^{1 / \beta}(\sup g)^{1 / \beta}\right)} \\
& =\lim _{t \rightarrow \infty} \int_{C_{1}^{+}[0,1]} \frac{1}{\sup \left(g f_{2}\right) \vee\left(t^{1 / \beta-1}(\sup g)^{1 / \beta}\right)} \mathrm{d} \Psi^{*}\left(f_{2}\right) \\
& =\int_{C_{1}^{+}[0,1]} \frac{1}{\sup \left(g f_{2}\right)} \mathrm{d} \Psi^{*}\left(f_{2}\right) .
\end{aligned}
$$

The last equation comes from the Lebesgue dominated convergence theorem and the fact that the last integral is finite. The proof of relation (5.10) follows the same line as in the two-dimensional case. The proposition is thus proved. Let

The last step in our construction is to assemble the constructed $\{W(s)\}_{s \in[0,1]}$ and $\{Z(s)\}_{s \in[0,1]}$.

$$
\{U(s)\}_{s \in[0,1]}:=\left\{W(s) \vee Z(s)^{\eta}\right\}_{s \in[0,1]},
$$

where the processes $\{W(s)\}_{s \in[0,1]}$ and $\{Z(s)\}_{s \in[0,1]}$ are independent. We show that $\{U(s)\}_{s \in[0,1]}$ fulfills all requirements in Theorem 5.2. To achieve this, we check three relations:

(a) by denoting the marginal distributions of $U(s)$ as $F_{S}(x)$, we have $1 /\left(1-F_{S}(t)\right) \sim t$ as $t \rightarrow \infty$

(b) for all $g \in C^{+}[0,1]$,

$$
\lim _{t \rightarrow \infty} t \mathrm{P}\left(\{U<t g\}^{\mathrm{c}}\right)=\int_{\bar{C}_{1}^{+}[0,1]} \sup _{s \in[0,1]} \frac{f_{1}}{g} \mathrm{~d} \rho\left(f_{1}\right) ;
$$

(c) for all $g \in C^{+}[0,1]$,

$$
\lim _{t \rightarrow \infty} t \mathrm{P}\left(\left\{U^{1 / \eta}>t g\right\}\right)=\int_{\bar{C}_{1}^{+}[0,1]} \frac{1}{\sup _{s \in[0,1]}\left(g f_{2}\right)} \mathrm{d} \Psi^{*}\left(f_{2}\right) .
$$

To prove (a) note that, for any $s \in[0,1]$ and $x>0$,

$$
\{U(s)>t x\}=\{W(s)>t x\} \cup\left\{Z(s)>(t x)^{1 / \eta}\right\} .
$$

Hence,

$$
t \mathrm{P}(W(s)>t x) \leq t \mathrm{P}(U(s)>t x) \leq t \mathrm{P}(W(s)>t x)+t \mathrm{P}\left(Z(s)>(t x)^{1 / \eta}\right) .
$$

Part (a) is proved by combining (5.7) and (5.10).

(b) For any $g \in C^{+}[0,1]$, we have

$$
\{U<\operatorname{tg}\}=\{W<\operatorname{tg}\} \cap\left\{Z^{\eta}<t g\right\} .
$$

Thus,

$$
\{U<t g\}^{\mathrm{c}}=\{W<t g\}^{\mathrm{c}} \cup\left\{Z^{\eta}<t g\right\}^{\mathrm{c}},
$$


which implies that

$$
t \mathrm{P}\left(\{W<t g\}^{\mathrm{c}}\right) \leq t \mathrm{P}\left(\{U<t g\}^{\mathrm{c}}\right) \leq t \mathrm{P}\left(\{W<t g\}^{\mathrm{c}}\right)+t \mathrm{P}\left(\left\{Z^{\eta}<t g\right\}^{\mathrm{c}}\right) .
$$

Considering (5.8), in order to prove (b), it is sufficient to prove that

$$
\lim _{t \rightarrow \infty} t \mathrm{P}\left(\left\{Z^{\eta}<t g\right\}^{\mathrm{c}}\right)=0 .
$$

By construction, $Z(s) \leq M^{\beta}$ for all $s \in[0,1]$. Hence,

$$
\left\{Z^{\eta}<t g\right\}^{\mathrm{c}} \subset\left\{M^{\beta \eta}>t \inf _{s \in[0,1]} g\right\},
$$

which implies that

$$
t \mathrm{P}\left(\left\{Z^{\eta}<t g\right\}^{\mathrm{c}}\right) \leq t \mathrm{P}\left(\left\{M^{\beta \eta}>t \inf g\right\}\right)=O\left(t^{1-1 /(\beta \eta)}\right) .
$$

From $\beta<1 / \eta$, we obtain $1-1 /(\beta \eta)<0$. This completes part (b).

(c) Given any $g \in C^{+}[0,1]$, on the one hand, we have

$$
\left\{U^{1 / \eta}>\operatorname{tg}\right\} \supset\{Z>\operatorname{tg}\},
$$

and, on the other hand, we have

$$
\begin{aligned}
\left\{U^{1 / \eta}>\right. & t g\} \\
\subset & \{Z>\operatorname{tg}\} \cup\left\{W^{1 / \eta}>\operatorname{tg}\right\} \\
& \cup\left\{\text { there exist } s_{1}, s_{2} \in[0,1] \text { such that } Z\left(s_{1}\right)>\operatorname{tg}\left(s_{1}\right) \text { and }\left(W\left(s_{2}\right)\right)^{1 / \eta}>\operatorname{tg}\left(s_{2}\right)\right\} \\
:= & S_{1} \cup S_{2} \cup S_{3} .
\end{aligned}
$$

Considering relation (5.9), in order to prove (c), it is sufficient to prove that $\lim _{t \rightarrow \infty} t \mathrm{P}\left(S_{j}\right)=0$ for $j=2,3$.

Considering restriction (5.3) on the spectral measure $\rho$, and the construction of $\{W(s)\}_{s \in[0,1]}$ in (5.6), we have $\mathrm{P}\left(\inf _{s \in[0,1]} Q_{0}(s)>0\right)=0$, which implies that

$$
\mathrm{P}\left(\inf _{s \in[0,1]} W(s)>0\right)=0 .
$$

Because $S_{2} \subset\left\{\inf _{s \in[0,1]} W(s)>0\right\}$, we obtain $\mathrm{P}\left(S_{2}\right)=0$.

The last step is on $S_{3}$. By construction, $Z(s) \leq M^{\beta}$ and $W(s) \leq M_{0}$ hold for all $s \in[0,1]$. The latter is implied by the fact that $\sup _{s \in[0,1]} Q_{0}(s)=1$. Therefore, we have

$$
S_{3} \subset\left\{M^{\beta}>t \inf g \text { and } M_{0}^{1 / \eta}>t \inf g\right\} .
$$

Since $M$ and $M_{0}$ are independent, we obtain

$$
\begin{aligned}
t \mathrm{P}\left(S_{3}\right) & \leq t \mathrm{P}\left(M>(t \inf g)^{1 / \beta}\right) \mathrm{P}\left(M_{0}>(t \text { inf } g)^{\eta}\right) \\
& =t O\left(t^{-1 / \beta}\right) O\left(t^{-\eta}\right) \\
& =O\left(t^{1-1 / \beta-\eta}\right) .
\end{aligned}
$$

From $1<\beta<1 / \eta$ and $\frac{1}{2}<\eta<1$, we obtain $1-1 / \beta-\eta<1-2 \eta<0$. It is thus proved that $\lim _{t \rightarrow \infty} t P\left(S_{3}\right)=0$, which completes the proof of part (c). 
The combination of (a) and (b) implies that the constructed process $\{U(s)\}_{s \in[0,1]}$ belongs to the domain of attraction of a max-stable process with extreme value dependence structure characterized by the spectral measure $\rho$. The combination of (a) and (c) implies that $\{U(s)\}_{s \in[0,1]}$ has extreme residual dependence characterized by the measure $\Psi^{*}$ with extreme residual coefficient $\eta$. Hence, Theorem 5.2 is proved.

Remark 5.2. Note that, for the stochastic process, the restriction on $\eta$ is that $0<\eta<1$, i.e. there is no lower bound restriction on $\eta$ in order to ensure the 'positively associated extremes'. This is in accordance with Remark 4.1 by considering that the number of dimensions tends to $\infty$.

Remark 5.3. The extreme value dependence implies that $\sup _{s \in[0,1]} \tilde{X}(s)$ has an extreme value index 1 , while the extreme residual dependence implies that inf $\sin _{s \in 1]} \tilde{X}(s)$ has an extreme value index $\eta$.

\section{Modeling systemic risk in a banking system}

It is often observed that banking crises are systemical, i.e. banks are likely to experience severe downside shocks simultaneously. This is called the systemic risk in banking system. One potential explanation of systemic risk is that banks share similar exposures to risk factors that are heavy tailed; see, e.g. de Vries (2005). This argument can be shown by the following simple model.

Consider a simple banking system with two banks $\left(B_{1}, B_{2}\right)$ holding a portfolio of risks on three independent risk factors $C, L_{1}$, and $L_{2}$. The losses of the two banks are given by

$$
B_{1}=C+L_{1}, \quad B_{2}=C+L_{2},
$$

where $\left(C, L_{1}, L_{2}\right)$ indicates the losses generated by the risk factors. Here $C$ is regarded as the common risk shared by $B_{1}$ and $B_{2}$, while $L_{1}$ and $L_{2}$ are idiosyncratic risks taken by individual banks, respectively. Suppose that $\left(C, L_{1}, L_{2}\right)$ are independent and follow heavytailed distributions. More specifically, we assume that $L_{i}$ has tail index $\alpha$ and $C$ has tail index $\beta$, i.e. as $x \rightarrow \infty$,

$$
\mathrm{P}\left(L_{i}>x\right) \sim \sigma_{L} x^{-\alpha} \quad \text { for } i=1,2, \quad \mathrm{P}(C>x) \sim \sigma_{C} x^{-\beta},
$$

where $\sigma_{L}, \sigma_{C}>0$ are the scales.

To examine the existence of the systemic risk, we may compare the probability of a systemic crisis with that of an individual crisis, i.e. calculating

$$
\kappa:=\lim _{t \rightarrow \infty} \frac{\mathrm{P}\left(B_{1}>t, B_{2}>t\right)}{\mathrm{P}\left(B_{2}>t\right)} .
$$

In de Vries (2005), it was shown that, when $\alpha=\beta, 0<\kappa<1$. Hence, when the systemic risk exists, the banking system is fragile.

Deviating from the assumption that $\alpha=\beta$ provides other possibilities for modeling different levels of systemic risk. The following theorem clarifies the different extreme dependence structure in different cases.

Theorem 6.1. Consider the simple model of a banking system in (6.1) and (6.2). Suppose that $\left(C, L_{1}, L_{2}\right)$ are all positive random variables. The following statements hold.

(a) When $\beta<\alpha,\left(B_{1}, B_{2}\right)$ are completely asymptotically dependent $(\kappa=1)$. 
(b) When $\beta=\alpha,\left(B_{1}, B_{2}\right)$ are partially asymptotically dependent $(0<\kappa<1)$.

(c) When $\alpha<\beta<2 \alpha,\left(B_{1}, B_{2}\right)$ are asymptotically independent $(\kappa=0)$; however, by defining $\tilde{B}_{i}=1 /\left(1-F_{i}\left(B_{i}\right)\right)$, where $F_{i}$ is the marginal distribution of $B_{i}$ for $i=1,2$, we have

$$
\lim _{t \rightarrow \infty} t^{\beta / \alpha} \mathrm{P}\left(\tilde{B}_{1}>t, \tilde{B}_{2}>t\right)=\sigma_{L}^{-\beta / \alpha} \sigma_{C} .
$$

(d) When $\beta>2 \alpha,\left(B_{1}, B_{2}\right)$ are asymptotically independent; moreover, with the same notation $\tilde{B}_{i}$ as in $(c)$,

$$
\lim _{t \rightarrow \infty} t^{2} \mathrm{P}\left(\tilde{B}_{1}>t, \tilde{B}_{2}>t\right)=1 .
$$

Proof. The proof of part (b) is given in de Vries (2005). It is not difficult to obtain $\kappa=$ $\sigma_{C} /\left(\sigma_{C}+\sigma_{L}\right)$. We now consider the other three cases.

(a) In this case, $\beta<\alpha$. The common risk has a heavier tail than the idiosyncratic risks. From the Feller theorem (see Feller (1971, Section VIII 8)), we have, as $t \rightarrow \infty$,

$$
\mathrm{P}\left(B_{i}>t\right) \sim \mathrm{P}(C>t) .
$$

On the other hand, for any $0<\varepsilon<1$, from

$$
\{C>t\} \subset\left\{B_{1}>t, B_{2}>t\right\} \subset\{C>(1-\varepsilon) t\} \cup\left\{L_{1}>\varepsilon t, L_{2}>\varepsilon t\right\},
$$

and the fact that $\mathrm{P}\left(L_{1}>\varepsilon t, L_{2}>\varepsilon t\right)=o(\mathrm{P}(C>t))$, we obtain

$$
1 \leq \liminf _{t \rightarrow \infty} \frac{\mathrm{P}\left(B_{1}>t, B_{2}>t\right)}{\mathrm{P}(C>t)} \leq \limsup _{t \rightarrow \infty} \frac{\mathrm{P}\left(B_{1}>t, B_{2}>t\right)}{\mathrm{P}(C>t)} \leq(1-\varepsilon)^{-\beta} .
$$

By taking $\varepsilon \rightarrow 0$, we obtain, as $t \rightarrow \infty$,

$$
\mathrm{P}\left(B_{1}>t, B_{2}>t\right) \sim \mathrm{P}(C>t) \sim \mathrm{P}\left(B_{2}>t\right) .
$$

Hence, $\kappa=1$.

(c) In this case, $\alpha<\beta<2 \alpha$. As $t \rightarrow \infty$,

$$
\mathrm{P}\left(B_{i}>t\right) \sim \mathrm{P}\left(L_{i}>t\right) \sim \sigma_{L} t^{-\alpha} .
$$

Hence, $1 /\left(1-F_{i}(t)\right) \sim t^{\alpha} / \sigma_{L}$ as $t \rightarrow \infty$.

Relation (6.4) implies that

$$
0 \leq \frac{\mathrm{P}\left(B_{1}>t, B_{2}>t\right)}{\mathrm{P}\left(L_{2}>t\right)} \leq \frac{\mathrm{P}(C>(1-\varepsilon) t)+\mathrm{P}\left(L_{1}>\varepsilon t, L_{2}>\varepsilon t\right)}{\mathrm{P}\left(L_{2}>t\right)} .
$$

Since $\beta>\alpha, \mathrm{P}(C>(1-\varepsilon) t)=o\left(\mathrm{P}\left(L_{2}>t\right)\right)$. Thus, we obtain

$$
\mathrm{P}\left(B_{1}>t, B_{2}>t\right)=o\left(\mathrm{P}\left(L_{2}>t\right)\right)=o\left(\mathrm{P}\left(B_{2}>t\right)\right),
$$

i.e. $\kappa=0$.

Next, from (6.4), note that

$$
\mathrm{P}\left(L_{1}>\varepsilon t, L_{2}>\varepsilon t\right)=\mathrm{P}\left(L_{1}>\varepsilon t\right) \mathrm{P}\left(L_{2}>\varepsilon t\right)=O\left(t^{-\alpha}\right) O\left(t^{-\alpha}\right)=o\left(t^{-\beta}\right)=o(\mathrm{P}(C>t)) .
$$


Similarly to case (b), we have $\mathrm{P}\left(B_{1}>t, B_{2}>t\right) \sim \mathrm{P}(C>t)$, which implies that

$$
\lim _{t \rightarrow \infty} t^{\beta} \mathrm{P}\left(B_{1}>t, B_{2}>t\right)=\sigma_{C} .
$$

From $1 /\left(1-F_{i}(t)\right) \sim t^{\alpha} / \sigma_{L}$ and $\tilde{B}_{i}=1 /\left(1-F_{i}\left(B_{i}\right)\right)$, we obtain

$$
\mathrm{P}\left(\tilde{B}_{i}>t\right) \sim \mathrm{P}\left(\frac{B_{i}^{\alpha}}{\sigma_{L}}>t\right) \quad \text { as } t \rightarrow \infty .
$$

A similar approximation holds for the joint probability $\mathrm{P}\left(\tilde{B}_{1}>t, \tilde{B}_{2}>t\right)$. A straightforward calculation yields

$$
\lim _{t \rightarrow \infty} t^{\beta / \alpha} \mathrm{P}\left(\tilde{B}_{1}>t, \tilde{B}_{2}>t\right)=\sigma_{L}^{-\beta / \alpha} \sigma_{C}
$$

(d) In this case, $\beta>2 \alpha$. The proof of $\kappa=0$ is similar to that in case (c). Analogous to the proofs of (b) and (c), from the relation

$$
\left\{L_{1}>t, L_{2}>t\right\} \subset\left\{B_{1}>t, B_{2}>t\right\} \subset\left\{L_{1}>(1-\varepsilon) t, L_{2}>(1-\varepsilon) t\right\} \cup\{C>\varepsilon t\},
$$

we obtain

$$
\mathrm{P}\left(B_{1}>t, B_{2}>t\right) \sim \mathrm{P}\left(L_{1}>t\right) \mathrm{P}\left(L_{2}>t\right) \sim \sigma_{L}^{2} t^{-2 \alpha}
$$

as $t \rightarrow \infty$. Together with the marginal tail distribution of $B_{i}$, it can be verified that

$$
\lim _{t \rightarrow \infty} t^{2} \mathrm{P}\left(\tilde{B}_{1}>t, \tilde{B}_{2}>t\right)=1 .
$$

Remark 6.1. In Theorem 6.1, we assumed that $C$ and $L_{i}$ are all positive random variables for simplicity. Such an assumption is not essential: assuming that the left tails of $C$ and $L_{i}$ are lighter, i.e. having higher tail indices, than the right tails is sufficient for obtaining the same result.

Remark 6.2. When $\left(B_{1}, B_{2}\right)$ are independent random variables, we have

$$
\lim _{t \rightarrow \infty} t^{2} \mathrm{P}\left(\tilde{B}_{1}>t, \tilde{B}_{2}>t\right)=\lim _{t \rightarrow \infty} t \mathrm{P}\left(\tilde{B}_{1}>t\right) t \mathrm{P}\left(\tilde{B}_{2}>t\right)=1 .
$$

Hence, Theorem 6.1(d) is comparable with the situation that systemic risk does not exist, while in Theorem 6.1(c), systemic risk still exists in the residual part.

Remark 6.3. In Theorem 6.1(c), relation (6.3) is comparable with (2.1) with $\eta=\alpha / \beta$. Formally, with a similar set manipulation procedure, we can obtain

$$
\begin{aligned}
\lim _{t \rightarrow \infty} & t^{\beta / \alpha} \mathrm{P}\left(\tilde{B}_{1}>t x_{1}, \tilde{B}_{2}>t x_{2}\right) \\
& =\lim _{t \rightarrow \infty} t^{\beta / \alpha} \mathrm{P}\left(B_{1}^{\alpha}>t \sigma_{L} x_{1}, B_{2}^{\alpha}>t \sigma_{L} x_{2}\right) \\
& =\lim _{t \rightarrow \infty} t^{\beta} \mathrm{P}\left(B_{1}>t \sigma_{L}^{1 / \alpha} x_{1}^{1 / \alpha}, B_{2}^{\alpha}>t \sigma_{L}^{1 / \alpha} x_{2}^{1 / \alpha}\right) \\
& =\lim _{t \rightarrow \infty} t^{\beta} \mathrm{P}\left(C>t\left(\sigma_{L} \max \left(x_{1}, x_{2}\right)\right)^{1 / \alpha}\right) \\
& =\frac{\sigma_{C}}{\left(\sigma_{L} \max \left(x_{1}, x_{2}\right)\right)^{\beta / \alpha}} .
\end{aligned}
$$

Hence, $\left(B_{1}, B_{2}\right)$ are extreme residual dependent with $\eta=\alpha / \beta$ and

$$
v^{*}\left\{(u, v): u>x_{1} \text { and } v>x_{2}\right\}=\frac{\sigma_{C}}{\sigma_{L}^{\beta / \alpha} \max \left(x_{1}, x_{2}\right)} \text {. }
$$


Such an extreme residual dependence structure is comparable with that in Example 3.1. Moreover, since $\alpha<\beta<2 \alpha$, we have $\frac{1}{2}<\eta<1$.

To summarize, when the common risk factor dominates the risks taken by the banks, the systemic risk is at the same level as the individual risk; hence, the system is in the most fragile situation. When the common risk factor and the idiosyncratic risks have comparable tails, the systemic risk exists, but at a level proportional to the individual risk; hence, the system is in a less fragile situation. When the idiosyncratic risks dominate but the common risk is still considerably heavy, i.e. $\alpha<\beta<2 \alpha$, the system is in the least fragile situation; however, the systemic risk still exists in the residual part. When the common risk has a much lighter tail than that of the idiosyncratic risk, i.e. $\beta>2 \alpha$, the systemic risk does not exist.

Our extended model captures not only asymptotically dependent cases but also asymptotically independent cases with dependence in the residual parts. Although local financial institutions from different economic regions, bearing their idiosyncratic risks as their major risks, do not exhibit strong fragility, a global crisis may still lead to a systemic crash due to the dependence in the residual parts. This explains the phenomenon observed in global crises: all financial institutions get a simultaneous systemic shock even though they may not be strongly linked. When modeling systemic risk within the banking system, it is necessary to take into account the dependence in residual parts, in order to avoid underestimation of systemic risk.

\section{Acknowledgement}

We would like to thank an anonymous referee for a careful reading and useful suggestions.

\section{References}

Coles, S. G. And TAwn, J. A. (1996). Modelling extremes of the areal rainfall process. J. R. Statist. Soc. B 58, $329-347$. De HaAn, L. And Ferreira, A. (2006). Extreme Value Theory. Springer, New York.

De HaAn, L. AND RESNick, S. I. (1977). Limit theory for multivariate sample extremes. Z. Wahrscheinlichkeitsth. 40, 317-337.

De HaAn, L. And Resnick, S. I. (1993). Estimating the limit distribution of multivariate extremes. Commun. Statist. Stoch. Models 9, 275-309.

De HaAn, L. And Stadtmüller, U. (1996). Generalized regular variation of second order. J. Austral. Math. Soc. 61, 381-395.

De Vries, C. G. (2005). The simple economics of bank fragility. J. Banking Finance 29, 803-825.

Draisma, G., Drees, H., Ferreira, A. AND De HaAn, L. (2004). Bivariate tail estimation: dependence in asymptotic independence. Bernoulli 10, 251-280.

Feller, W. (1971). An Introduction to Probability Theory and Its Applications, Vol. 2, 2nd edn. John Wiley, New York.

Ferreira, A., De HaAn, L. AND ZHOU, C. (2009). Exceedance probability of the integral of a stochastic process. Unpublished manuscript.

Geffroy, J. (1958). Contribution à la théorie des valeurs extrêmes. Publ. Inst. Statist. Univ. Paris 7, $37-121$.

Giné, E., Hahn, M. G. And Vatan, P. (1990). Max-infinitely divisible and max-stable sample continuous processes. Prob. Theory Relat. Fields 87, 139-165.

Heffernan, J. E. ANd Resnick, S. I. (2005). Hidden regular variation and the rank transform. Adv. Appl. Prob. 37, 393-414.

LEDFORD, A. W. AND TAWN, J. A. (1996). Statistics for near independence in multivariate extreme values. Biometrika 83, 169-187.

Ledford, A. W. And TAwn, J. A. (1997). Modelling dependence within joint tail regions. J. R. Statist. Soc. B 59, 475-499.

Ledford, A. W. And Tawn, J. A. (1998). Concomitant tail behaviour for extremes. Adv. Appl. Prob. 30, 197-215.

Ledford, A. W. And Tawn, J. A. (2003). Diagnostics for dependence within time series extremes. J. R. Statist. Soc. B 65, 521-543.

MaUlik, K. AND Resnick, S. (2004). Characterizations and examples of hidden regular variation. Extremes 7, 31-67. 
Poon, S.-H., Rockinger, M. And TAwn, J. (2004). Extreme value dependence in financial markets: diagnostics, models, and financial implications. Rev. Financial Studies 17, 581-610.

Ramos, A. ANd LedFord, A. (2009). A new class of models for bivariate joint tails. J. R. Statist. Soc. B 71, $219-241$.

RESNICK, S. (2002). Hidden regular variation, second order regular variation and asymptotic independence. Extremes 5, 303-336.

SibuYa, M. (1960). Bivariate extreme statistics. I. Ann. Inst. Statist. Math. 11, 195-210. 